\title{
The Lyman Alpha Reference Sample: III. Properties of the Neutral ISM from GBT and VLA Observations
}

\author{
Stephen A. Pardy \\ Department of Astronomy, University of Wisconsin, 475 North Charter Street, Madison, \\ WI 53706, USA \\ Department of Physics 85 Astronomy, Macalester College, 1600 Grand Avenue, Saint Paul, \\ $M N$ 55105, USA \\ spardy@astro.wisc.edu \\ John M. Cannon \\ Department of Physics 85 Astronomy, Macalester College, 1600 Grand Avenue, Saint Paul, \\ MN 55105 \\ jcannon@macalester.edu \\ Göran Östlin \\ Department of Astronomy, Oskar Klein Centre, Stockholm University, AlbaNova University \\ Centre, SE-106 91 Stockholm, Sweden \\ ostlin@astro.su.se \\ Matthew Hayes \\ Department of Astronomy, Oskar Klein Centre, Stockholm University, AlbaNova University \\ Centre, SE-106 91 Stockholm, Sweden \\ matthew.hayes@astro.su.se \\ Thøger Rivera-Thorsen \\ Department of Astronomy, Oskar Klein Centre, Stockholm University, AlbaNova University \\ Centre, SE-106 91 Stockholm, Sweden \\ trive@astro.su.se \\ Andreas Sandberg \\ Department of Astronomy, Oskar Klein Centre, Stockholm University, AlbaNova University \\ Centre, SE-106 91 Stockholm, Sweden
}


sandberg@astro.su.se

Angela Adamo

Max Planck Institute for Astronomy, Königstuhl 17, D-69117 Heidelberg, Germany

adamo@mpia.de

Emily Freeland

Department of Astronomy, Oskar Klein Centre, Stockholm University, AlbaNova University Centre, SE-106 91 Stockholm, Sweden

emily.freeland@astro.su.se

E. Christian Herenz

Leibniz-Institut für Astrophysik (AIP), An der Sternwarte 16, D-14482 Potsdam, Germany.

cherenz@aip.de

Lucia Guaita

Department of Astronomy, Oskar Klein Centre, Stockholm University, AlbaNova University Centre, SE-106 91 Stockholm, Sweden

lguai@astro.su.se

Daniel Kunth

Institut d'Astrophysique de Paris, 98bis, bd Arago, 75014 Paris, France

kunth@iap.fr

Peter Laursen

Dark Cosmology Centre, Niels Bohr Institute, University of Copenhagen, Juliane Maries Vej 30, 2100 Copenhagen, Denmark

pela@dark-cosmology.dk

J. M. Mas-Hesse

Centro de Astrobiología (CSIC-INTA), Madrid, Spain

mm@cab.inta-csic.es 
Jens Melinder

Department of Astronomy, Oskar Klein Centre, Stockholm University, AlbaNova University Centre, SE-106 91 Stockholm, Sweden

$$
\text { jens@astro.su.se }
$$

Ivana Orlitová

Geneva Observatory, University of Geneva, 51 Chemin des Maillettes, CH-1290 Versoix, Switzerland

Astronomical Institute, Academy of Sciences of the Czech Republic, Bocni II, CZ-14131 Prague, Czech Republic

ivana.orlitova@unige.ch

\section{Héctor Otí-Floranes}

Instituto de Astronomía, Universidad Nacional Autónoma de México, Apdo. Postal 106, Ensenada B. C. 22800 Mexico

$$
\text { otih@astrosen.unam.mx }
$$

Johannes Puschnig

Department of Astronomy, Oskar Klein Centre, Stockholm University, AlbaNova University Centre, SE-106 91 Stockholm, Sweden

$$
\text { johannes.puschnig@astro.su.se }
$$

Daniel Schaerer

Geneva Observatory, University of Geneva, 51, Ch. des Maillettes, CH-1290 Versoix, Switzerland

Université de Toulouse; UPS-OMP; IRAP; Toulouse, France

$$
\text { daniel.schaerer@obs. unige.ch }
$$

Anne Verhamme

Geneva Observatory, University of Geneva, 51, Ch. des Maillettes, CH-1290 Versoix, Switzerland 
anne.verhamme@unige.ch

\begin{abstract}
We present new H I imaging and spectroscopy of the 14 UV-selected starforming galaxies in the Lyman Alpha Reference Sample (LARS), aimed for a detailed study of the processes governing the production, propagation, and escape of Ly $\alpha$ photons. New H I spectroscopy, obtained with the 100m Green Bank Telescope (GBT), robustly detects the H I spectral line in 11 of the 14 observed LARS galaxies (although the profiles of two of the galaxies are likely confused by other sources within the GBT beam); the three highest redshift galaxies are not detected at our current sensitivity limits. The GBT profiles are used to derive fundamental H I line properties of the LARS galaxies. We also present new pilot $\mathrm{H}$ I spectral line imaging of 5 of the LARS galaxies obtained with the Karl G. Jansky Very Large Array (VLA). This imaging localizes the H I gas and provides a measurement of the total H I mass in each galaxy. In one system, LARS 03 (UGC 8335 or Arp 238), VLA observations reveal an enormous tidal structure that extends over $160 \mathrm{kpc}$ from the main interacting systems and that contains $>10^{9} \mathrm{M}_{\odot}$ of H I. We compare various H I properties with global Ly $\alpha$ quantities derived from HST measurements. The measurements of the Ly $\alpha$ escape fraction are coupled with the new direct measurements of H I mass and significantly disturbed H I velocities. Our robustly detected sample reveals that both total H I mass and linewidth are tentatively correlated with key Ly $\alpha$ tracers. Further, on global scales, these data support a complex coupling between Ly $\alpha$ propagation and the H I properties of the surrounding medium.
\end{abstract}

Subject headings: galaxies: ISM — galaxies: starburst — galaxies: kinematics and dynamics — radio lines: galaxies

\title{
1. Introduction
}

The Lyman-alpha emission line (Ly $\alpha)$ at $1216 \AA$ fulfills several extremely important roles in observations of the high-redshift $(z)$ Universe. Recombination nebulae re-process $\sim 1 / 3$ of the raw ionizing power of hot stars into a single emission line which acts as a luminous spectral beacon by which to identify galaxies at the highest redshifts (see, e.g., Hayes et al. 2010 and references therein). Further, at the highest redshift it provides the potential for 
the all-important spectroscopic confirmation of distant galaxies selected by other methods, transforming the status of candidates to objects with secure redshifts (e.g., Stark et al. 2010).

$\operatorname{Ly} \alpha$ is a resonance line and is readily scattered by neutral hydrogen atoms. This scattering may occur in the interstellar medium (ISM) of the galaxies themselves, or in the circumgalactic medium (CGM) that immediately surrounds them. Scattering in the CGM can lead to extended Ly $\alpha$ structures such as those found in high-z Ly $\alpha$ emitters (LAE) by Steidel et al. (2011) and Zheng et al. (2011) among others. Inside the ISM, the radiative transport of Ly $\alpha$ depends strongly on dust extinction (Atek et al. 2014). This dependence, however, shows large scatter due to the complicated resonant scattering of Ly $\alpha$, in which the visibility of the line is influenced by a large number of factors, including dust content (Charlot \& Fall 1993; Verhamme et al. 2008; Atek et al. 2009; Hayes et al. 2010), dust geometry (Scarlata et al. 2009), neutral gas content and kinematics (Kunth et al. 1998; Mas-Hesse et al. 2003; Cannon et al. 2004), and gas geometry (Neufeld 1991; Giavalisco et al. 1996; Hansen \& Oh 2006; Laursen et al. 2013; Duval et al. 2014). Taken together, these myriad factors indicate that the true total escape fraction of Ly $\alpha$ photons $\left(f_{\mathrm{esc}}^{\mathrm{Ly} \alpha}\right.$; defined as the ratio of observed to intrinsic Ly $\alpha$ luminosity; Hayes et al. 2005, Hayes et al. 2013), is still poorly understood. Thus, photometric measurements of Ly $\alpha$ will reflect the underlying properties of galaxies only in the very broadest statistical sense.

The only way to remedy this complicated situation is to study the Ly $\alpha$ emission line in a sample of star-forming galaxies on a spatially resolved basis. From the UV perspective, obtaining Ly $\alpha$ images in the nearby Universe is expensive and requires space-based platforms. To this end, our team has acquired data from the Hubble Space Telescope (HST) to produce Ly $\alpha$ images of a statistically selected sample of star-forming galaxies in the low- $z$ Universe (see Östlin et al. 2014); details about the image processing methods can be found in Hayes et al. (2009). This program, called the "Lyman Alpha Reference Sample" ("LARS"), includes HST Cycle 18 imaging and Cycle 19 spectroscopic observations of 14 galaxies. These HST datasets form the backbone of a comprehensive multi-wavelength observational campaign that will allow great strides forward in our understanding of Ly $\alpha$ radiative transport. Since the LARS sample is selected on UV luminosity and $\mathrm{H} \alpha$ equivalent width, and not on Ly $\alpha$ characteristics, the composite sample was designed to overlap with both high- $z$ and low- $z$ star-forming galaxies. LARS will form a critical local interpretive benchmark for studies of high- $z$ galaxies for decades to come, and is well placed to take advantage of the remaining HST lifetime and motivate future observations with JWST.

The LARS program (including sample selection) is described in detail in Östlin et al. (2014) and Hayes et al. (2014), hereafter referred to as Papers I and II respectively. Preliminary results derived from the Ly $\alpha$ images of the LARS galaxies are presented in Hayes 
et al. (2013). That work showed that in many LARS galaxies, Ly $\alpha$ emission is prominent on physical scales that exceed those of both the massive stellar populations and the star formation regions that give rise to the Ly $\alpha$ photons; this trend is very prominent in the panels of Figures 1, 2, and 3, which show optical and UV images of the LARS galaxies. This may point towards Ly $\alpha$ photons being resonantly scattered to large radii in most of the LARS galaxies, although other possibilities have been proposed, such as ionization caused by hot plasma (Otí-Floranes et al. 2012). It is likely that multiple of the aforementioned physical properties (dust content and geometry, neutral gas content, kinematics, geometry) are facilitating this efficient resonant scattering.

In order to decouple the effects of dust and neutral gas kinematics and densities, the Ly $\alpha$ images must be compared with spatially resolved information about the neutral ISM. While UV and optical absorption line spectroscopy offers one avenue to address this issue, such data are limited to those regions in the foreground of only the brightest continuum sources (usually the emission line nebulae themselves). While our team is pursuing such analyses (see Rivera-Thorsen et al., in preparation, and Ly $\alpha$ profile modeling from Orlitova et al., in preparation), these avenues should be complemented by direct probes of the neutral interstellar medium in all regions of the LARS galaxies. To this end, in this manuscript we begin a detailed exploration of the neutral hydrogen in the LARS galaxies by presenting new single-dish HI spectroscopy and spatially resolved interferometric H I imaging. We use these data to derive global properties and to study the properties of the H I gas on bulk scales.

Previous spatially resolved H I observations of galaxies in which Ly $\alpha$ radiative transport has been studied in detail are few. While the sample of systems with spectroscopic information about Ly $\alpha$ is robust, there are comparatively few systems that have direct Ly $\alpha$ imaging (see the discussion in Hayes et al. 2005, Hayes et al. 2009, Östlin et al. 2009, and references therein). For those systems that do, there has, until the present work, been no systematic investigation of the H I on a spatially resolved basis; only a few selected systems have detailed H I studies in the literature.

Cannon et al. (2004) presented H I observations of two of the most well-studied and nearby Ly $\alpha$-emitting galaxies in the local Universe, ESO 338-IG 004 (Tol 1924-416) and IRAS $08339+6517$. In each galaxy, extended neutral gas connects the target and nearby neighbors, suggesting that interactions have played an important role in triggering the massive starbursts in ESO 338-IG 004 and IRAS 08339+6517. The close proximity of the companions suggests that the interactions were recent. Since both primary systems are Ly $\alpha$ emitters, these data support two possible interpretations of Ly $\alpha$ escape from starburst galaxies: either a) the bulk ISM kinematics provides the means of escape for Ly $\alpha$ photons by shifting $\mathrm{H} \mathrm{I}$ atoms out of resonance, or b) the H I is sufficiently clumpy on scales below our reso- 
lution to allow for efficient Ly $\alpha$ escape. These interpretations are further supported by H I (Bergvall \& Jorsater 1988) and Ly $\alpha$ observations (Kunth et al. 1998) of ESO400-G43.

In this work, we begin to extend this type of analysis to the entire LARS sample. Basic properties of the LARS galaxies are summarized in Table 1, Figures 1, 2, and 3 show optical and UV images of the 14 LARS galaxies, with the left and right columns displaying the color HST images and the SDSS color images respectively. The right column includes the immediate surroundings, while the left column has smaller fields of view, and are scaled to show detail in the galaxies.

We organize this manuscript as follows. In $\S 2$ we describe the data acquisition, reduction, and analysis. $\S 3$ presents the single-dish H I profiles of the 14 LARS galaxies, while $\S 4$ presents spatially resolved H I images of 5 of the 14 LARS systems. We discuss each system individually in $\S 5$, and discuss various correlations between galaxy global properties in $\S 6$. We draw our conclusions in $\S 7$. Throughout this paper we adopt the value of $\mathrm{H}_{0}=$ $70 \pm 1.4 \mathrm{~km} \mathrm{~s}^{-1} \mathrm{Mpc}^{-1}$ from the averaged WMAP 7 year data as presented in Komatsu et al. (2011).

\section{Observations, Data Reduction and Analysis}

\subsection{GBT Data}

We obtained data from the National Radio Astronomy Observatory 100m Robert C. Byrd Green Bank Telescope (GBT ${ }^{1}$ ) under program GBT/11A-057 (Legacy ID QO13; P.I. Östlin). Data were acquired in four observing sessions in March and April of 2011. Position switching observations were performed for all sources; 32,768 channels over the $12.5 \mathrm{MHz}$ of total bandwidth produce a spectral resolution of $381.469 \mathrm{~Hz}\left(0.08 \mathrm{~km} \mathrm{~s}^{-1} \mathrm{ch}^{-1}\right)$.

All reductions were performed in the IDL environment ${ }^{2}$, using the GBTIDL package designed at NRAO. We first imported the raw data for each galaxy into GBTIDL using standard averaging techniques, with zenith opacity coming from atmospheric data from the NRAO CLEO weather system. The data was moderately contaminated by terrestrial radio frequency interference (RFI); bad data were identified by examining individual spectra, blanked, and interpolated.

\footnotetext{
${ }^{1}$ The National Radio Astronomy Observatory is a facility of the National Science Foundation operated under cooperative agreement by Associated Universities, Inc.

${ }^{2}$ Exelis Visual Information Solutions, Boulder, Colorado
} 
The standard position switching algorithms in GBTIDL were used to produce a combined spectrum for each galaxy. To increase the Signal to Noise Ratio (SNR) of the observations we first averaged the reference spectrum by 16 channels. This procedure can have a negative effect on the baseline shape, increasing the order of the polynomial (often 3rd order) that we used to fit and subtract the continuum (see Table 2 for the order of the fit for each galaxy). The SNR was quite low on most of the LARS galaxies and we thus smoothed our spectrum by 256 channels resulting in a velocity resolution of $\sim 20 \mathrm{~km} \mathrm{~s}^{-1}$. Flux and width measurements were taken on this smoothed profile. Two regions were selected by hand for each galaxy: the first around the profile, and the second in an area free from RFI (used as an RMS noise region).

The redshifts of the LARS galaxies (see table 1) place some of them in proximity to a bandpass filter in the 1.2-1.3 GHz region. This bandpass filter is designed to shield against a local radar signal, but contributes higher than usual system temperature $\left(\mathrm{T}_{\text {sys }}\right)$ values (and therefore system noise) to calibrated data in this frequency range. Further, the $\mathrm{T}_{\text {sys }}$ values fluctuated markedly from one spectral scan to the next. The affected galaxies were all non detections (LARS 12, LARS 13, and LARS 14) and have markedly higher $\mathrm{T}_{\text {sys }}$ values (in particular LARS 13) than would otherwise be expected. It should also be noted that these three galaxies are at the largest redshifts in our sample.

Systematically measuring the flux and width of irregular, low SNR, H I spectra can be challenging. To do so, we have modified previously well known measurement techniques and find that they are robust over the range of SNR and profile shapes in our data. Our measurement routines take as inputs the smoothed spectrum and the region suspected of containing the galaxy. In our initial tests of archival data for NGC 5291 (available in the distribution of GBTIDL), we found that hand selecting regions of the profile could add additional bias, causing the width and flux measurements to vary by $2-5 \%$. Instead, we opted to use $\chi^{2}$ minimization techniques to fit Gaussian components (a single component fit for galaxies with a single peak and a multi component fit for double-horned profiles) to the smoothed spectrum. These components were used to guide later analysis and ensured repeatability in our results even with low SNR. These Gaussian fits serve only to guide analysis, however, and the flux and width measurements follow the methodology described in Springob et al. (2005). This method fits lines to the profile in the region over which it varies the most (here between $15 \%$ and $85 \%$ of the peak value) and measures width properties from these fits. Springob et al. (2005) found that this reduced the dependence on noise on their measurements versus direct measurements. We modified this method to respond better to low SNR values and to handle single peaked data more accurately. We consider this method to be the "best representation" of the width measurements for these spectra, although it is not the only method (e.g., the busy function; Westmeier et al. 2014). We recorded two SNRs 
based on the peak-to-rms ratio and on the integrated flux to integrated rms ratio.

The flux accuracy of GBT data using standard reduction techniques is quoted as $10 \%$ in the GBTIDL calibration guide. The primary error terms are the RMS fluctuations of the spectra in the region around the target and the subjectivity of selecting the baseline regions and RMS/profile regions. In testing it was seen that increasing the order of the baseline generally led to increasing error. We thus adopt a $10 \%$ flux error for "good" profiles and a conservative $20 \%$ for "irregular" profiles.

Special attention was paid to the error terms presented in Springob et al. (2005) as modified from Schneider et al. (1990). The error term given there adds an error associated with the quality of the baseline fit. Because we have assumed a separate baseline error, we remove this term to get a more or less standard error term:

$$
\epsilon_{S}^{\text {stat }}=\operatorname{rms} \sqrt{W \Delta V}
$$

where $\epsilon_{S}^{\text {stat }}$ is the noise in units of $\mathrm{Jy} \mathrm{km} \mathrm{s}^{-1}$, and $\mathrm{W}$ is the area of integration for the profile. The width at $50 \%$ of the maximum value $\left(\mathrm{W}_{50}\right)$ results reported are highly dependent on the accuracy of the method described in Springob et al. (2005) which, as mentioned in the above discussion, breaks down at lower SNRs.

The error for the width and systemic velocity measurements is based on the equations found in Schneider et al. (1990).

$$
\sigma V=1.5\left(W_{20}-W_{50}\right)(\mathrm{SNR})^{-1}
$$

This equation is doubled to give the error of the width measurements $W_{20}$ and $W_{50}$, which refer to the width at $20 \%$ and $50 \%$ of the maximum, respectively.

\subsection{VLA Data}

We obtained D-configuration Very Large Array $\left.\right|^{3}$ H I spectral line imaging of five LARS galaxies under program VLA/13A-181 (Legacy ID AC 1123; P.I. Cannon). These data were obtained with a standard WIDAR correlator configuration that provides $16 \mathrm{MHz}$ of total bandwidth. 1,024 channels deliver a native spectral resolution of $3.3 \mathrm{~km} \mathrm{~s}^{-1}$. The data were acquired in March and April of 2013, during seven observing sessions; details are summarized in Table 3 ,

\footnotetext{
${ }^{3}$ The National Radio Astronomy Observatory is a facility of the National Science Foundation operated under cooperative agreement by Associated Universities, Inc.
} 
Data reductions followed standard prescriptions in CASA 4 and in AIPS5. The wide bandwidth and frequency range of these observations resulted in a significant amount of radio frequency interference that was removed from the data. Bandpass and flux calibrations were derived using either 3C147 or 3C286 (see Table 3). The gains and phases were then calibrated using observations of the phase calibrators, which were typically separated by $\sim 20$ minutes of on-source integration time. The wide bandwidth allowed for hundreds of channels of line-free continuum to be fit and subtracted. For LARS 02 and LARS 09, which each had two separate observing sessions, we combined the separate observations into a single dataset for each galaxy.

The calibrated data were then inverted and cleaned using the CLEAN algorithms in CASA. To maximize SNR, the datasets for LARS 04 and LARS 08 were spectrally smoothed to a final velocity resolution of $10 \mathrm{~km} \mathrm{~s}^{-1}$. A Gaussian UVTAPER was applied to produce cubes with beam sizes of $\sim 1^{\prime}$; the resulting cubes were then convolved to circular beam sizes. The beam sizes and rms noises of the final datacubes are summarized in Table 4 .

Moment maps were derived using standard blanking procedures. First, each datacube was spatially smoothed to a circular beam size that is larger than the original. The resulting smoothed cube was then blanked at the $2.5 \sigma$ level, where $\sigma$ is the rms noise in line-free channels of the cube. This blanked cube is then inspected by hand, and only features that are spatially coincident across two or more consecutive channels are deemed to be real emission and kept in the final blanked cube. This cube is then used as a blanking mask against the original, unconvolved cube. Moment maps are then derived using standard procedures. We assume a $10 \%$ uncertainty on the overall calibration and absolute flux scale of the VLA images.

It is important to emphasize that all of the LARS galaxies are at distances of more than $100 \mathrm{Mpc}\left(\mathrm{z}_{\mathrm{opt}} \geq 0.028\right.$; see Table 1); even with angular resolutions that are exquisite for H I work (e.g., $5^{\prime \prime}=2.4 \mathrm{kpc}$ at a distance of $100 \mathrm{Mpc}$ ), we are sensitive only to the bulk $\mathrm{H} \mathrm{I}$ properties of the neutral gas in these galaxies. As such, the VLA images presented here are used only to study the global morphology and large-scale H I kinematics. A detailed study of the $\mathrm{H}$ I on finer physical scales will require deep observations in more extended array configurations.

\footnotetext{
${ }^{4}$ Common Astronomy Software Application (CASA) is developed by the NRAO.

${ }^{5}$ Astronomical Image Processing System (AIPS) is developed by the NRAO.
} 


\section{3. $\quad$ Ly $\alpha$ Data}

We use global Ly $\alpha$ data for all 14 LARS galaxies as presented in Paper I and Paper II. The HST data were obtained with the Wide Field Camera 3 (WFC3) and Advanced Camera for Surveys (ACS) using $\mathrm{H} \alpha$ and $\mathrm{H} \beta$ filters, and a combination of $\mathrm{U}, \mathrm{B}$, and $\mathrm{I}$ bands for $\mathrm{FUV}$ continuum imaging. We also acquired data with the Solar Blind Channel using a bandpass designed to isolate Ly $\alpha$ emission.

We reduced imaging data using MULTIDRIZZLE ${ }^{6}$ in the standard HST pipelines and used the Lyman-alpha extraction Software (LaXs) (Hayes et al. 2009) to continuum subtract the Ly $\alpha$ UV data. We refer the reader to Paper I and II for details of this process. The final global properties (see Paper I and table 5) are measured within apertures that correspond to twice the Petrosian radius $(\eta=0.2$; Petrosian 1976). This reduces the effect of noise on the measurements at large radii.

\section{GBT HI Global Profiles}

Presented in Figure 4 are the fully smoothed and reduced GBT H I profiles of the LARS galaxies. Plotted over these are the Gaussian fits in gray, solid lines in red showing the sides of the profile used in the peak $\mathrm{W}_{50}$ calculations, and the peak $\mathrm{W}_{50}$ line itself shown as a horizontal blue dotted line. A discussion of each galaxy is included below.

The observed properties from our GBT observations of each galaxy can be found in Table 2 along with properties derived from these using methods described below. The distances of each galaxy are calculated from the standard LARS luminosity distances. The $\mathrm{H}$ I mass in units of $\mathrm{M}_{\odot}$ is calculated via $\mathrm{M}_{\mathrm{HI}}=2.36 \times 10^{5} \mathrm{D}^{2} \mathrm{~S}_{\mathrm{HI}}$ where $\mathrm{D}$ is the distance in $\mathrm{Mpc}$ and $\mathrm{S}_{\mathrm{HI}}$ is the $\mathrm{H}$ I flux integral in units of $\mathrm{Jy} \mathrm{km} \mathrm{s}^{-1}$. We will discuss the mass value for each galaxy in section 5. Overall we find that our detections and mass estimates show a strong dependence on distance.

It is important to emphasize that the $8^{\prime}$ primary beam of the GBT at $21 \mathrm{~cm}$ is sensitive to all H I-bearing objects in the observed frequency range. Even for the closest LARS galaxy (LARS 01; $\mathrm{D}=120 \pm 2.4 \mathrm{Mpc}$ ), this $8^{\prime}$ beam subtends a projected circular area that is nearly $280 \mathrm{kpc}$ in diameter; the potential for contamination in the GBT beam is significant. We thus examined SDSS images of the area surrounding each LARS galaxy for nearby objects. Objects with spectroscopic data available from SDSS that might have contributed to the

\footnotetext{
${ }^{6}$ Fruchter, A. and Sosey, M. et al. 2009, "The MultiDrizzle Handbook", version 3.0, (Baltimore, STScI)
} 
GBT flux are noted below. These objects have velocities within $300 \mathrm{~km} \mathrm{~s}^{-1}$ of the target LARS galaxy and are within the $8^{\prime}$ primary beamsize of the GBT. Optical images of the fields that contain possible confusing sources (LARS 06 and LARS 11) are shown in Figures 5 and 6; these two sources are discussed in more detail in $\S 5$. We used the SDSS g band to convert to luminosity $\mathrm{L}_{\mathrm{g}}$, which we compared with a $\log \left(\mathrm{M}_{\mathrm{HI}} / \mathrm{L}_{\mathrm{B}}\right)$ value of -0.5 for late type galaxies found in (Haynes \& Giovanelli 1984). The masses of potential contamination galaxies are computed from this ratio. We also use the 22.2 magnitude limit for the SDSS to determine the possible contributions from optically dim sources of contamination. For our closest nine galaxies this is roughly $10^{6} \mathrm{M}_{\odot}$ of $\mathrm{H} \mathrm{I}$; this increases to $10^{8}$ for our furthest two systems. This information is also presented in Table 2.

The profiles in Figure 4 show a variety of H I structure. Some galaxies are simple single peak profiles (e.g, LARS 04, LARS 09), while others show complex and asymmetric profiles (e.g., LARS 01, LARS 03). Still other systems have H I spectra that are almost certainly confused; the classic double-horn profile of LARS 06 is likely due to the contribution of UGC 10028, a large spiral galaxy located only $1^{\prime}$ to the southeast of LARS 06 (see Figure 5).

Various observed properties of each GBT H I profile, and the quantities derived from these, are tabulated in Table 22 column 1 identifies the galaxy number; column 2 is the systemic velocity in $\mathrm{km} \mathrm{s}^{-1}$; column 3 is the linewidth at $50 \%$ in $\mathrm{kms}^{-1}$; column 4 is the linewidth at $20 \%$ in $\mathrm{km} \mathrm{s}^{-1}$; column 5 is the velocity offset between the optical velocity and the H I central velocity; column 6 is the the single-dish integrated flux in Jy km s${ }^{-1}$; column 7 is the mass from the single-dish measurements in units of $10^{9} \mathrm{M}_{\odot}$; column 8 is the SNR from the integrated flux and the peak; column 9 is the rms noise value of the GBT in units of mJy; column 10 gives the baseline polynomial fit used in the calibration of the GBT spectrum; column 11 is the classification of the profile ('S' for single-horned, 'D' for double-horned, ' $N$ ' for no detection, 'I' for highly irregular shapes, or the presence of RFI near the signal region, and ' $\mathrm{C}$ ' for confusion due to known nearby galaxies.)

\section{VLA H I Images}

The H I moment zero (representing H I mass surface density) and moment one (representing intensity-weighted velocity) images of the 5 LARS galaxies observed in program 13A-181 are presented in Figures 7, 8, 9, 10, and 11. In panel (a) of each figure, a Digitized Sky Survey image is overlaid with contours of H I mass surface density (individual contour levels are provided in the caption to each figure). The beam size is shown by a blue circle, while a red square shows the location of the HST pointing for which we have UV imaging (see discussion in Paper I and II). In panel (b) of each figure, the H I moment one image 
(representing intensity weighted velocity) is presented in a rainbow color format; the velocity scale of each galaxy is shown by a colorbar.

Based on the distances and optical angular sizes of the LARS galaxies (of order $1^{\prime}$ or less; see Figures 1, 2, and 3), one would expect the H I to be distributed on similar angular scales. At the resolution of these data (beam sizes between $59^{\prime \prime}$ and $72^{\prime \prime}$ ), the LARS galaxies would thus appear as unresolved sources in the H I data cubes. As we discuss below, three of the LARS galaxies have H I morphologies consistent with this interpretation. Surprisingly, two of the sources have H I distributions that largely exceed the beam size; we discuss these sources in detail below.

It is important to stress that even in cases where galaxies are unresolved by an $\mathrm{H} \mathrm{I}$ beam, one can still extract bulk characteristics of the neutral ISM because of the spectrally resolved nature of the data. Detailed kinematic analyses (e.g., rotation curve work or spatially resolved position-velocity diagrams) will be unavailable in such cases. However, within the spatial and spectral resolution elements of the data, one can derive bulk constraints on the motions of the $\mathrm{H}$ I gas.

We use the VLA H I images of the 5 LARS galaxies presented here to constrain two critical properties. First, the H I data allow us to localize the H I associated with the LARS galaxy. This in turn allows us to accurately measure the total H I mass of each system. These interferometric measurements often recover less of the H I flux, and therefore mass, than the GBT measurements. We attribute this to the low SNR of H I in these galaxies, the possible presence of low surface brightness H I features missed by the VLA in extended regions or velocity space, and flux contamination from other galaxies, although we suspect that this is only relevant in the cases marked as confused.

It is interesting to note that at the distances of the LARS galaxies, interferometric observations are perhaps better suited to determine the $\mathrm{H}$ I mass than single-dish observations (for example, the $60^{\prime \prime}$ characteristic resolution of the present data are about a factor of three better than the H I beam of Arecibo, the largest single-dish radio telescope in the world, and a factor of eight better than our GBT resolution), simply because of the potential for contamination in the single-dish beam. Second, the present data are sensitive to large-scale distributions of $\mathrm{H} \mathrm{I}$ gas, some of which is expected to be tidal in origin based on the optical morphologies of the LARS galaxies alone (see, e.g., Figures 1, 2, and 3).

In Table 4 we present VLA H I quantities for our five targeted galaxies derived in the same method as described above for the GBT data. Column 1 identifies the galaxy number; column 2 is the systemic velocity in $\mathrm{km} \mathrm{s}^{-1}$; column 3 is the linewidth at $50 \%$ in $\mathrm{km} \mathrm{s}^{-1}$; column 4 is the linewidth at $20 \%$ in $\mathrm{km} \mathrm{s}^{-1}$; column 5 is the velocity offset between the 
optical velocity and the $\mathrm{H}$ I central velocity; column 6 is the the interferometric integrated flux in Jy km s${ }^{-1}$; column 7 is the mass in units of $10^{9} \mathrm{M}_{\odot}$; column 8 is the rms noise value in units of $\mathrm{mJy} /$ Beam; column 9 is the median global column density of $\mathrm{H}$ I from a column density map convolved with the beam size given in $10^{19} \mathrm{~cm}^{-2}$.

\section{Discussion of Individual Galaxies}

We now present discussion of each LARS target based on our new H I data and on the HST imaging presented in Hayes et al. (2013), Paper I, and Paper II.

\subsection{LARS 01}

LARS 01 is a strong Ly $\alpha$ emitter, with a global EW of $46 \AA$ (Paper I) and a moderate star formation rate (a few $\mathrm{M}_{\odot} \mathrm{yr}^{-1}$ ). The single-dish $\mathrm{H}$ I observations (see Figure 4 ) reveal an asymmetric double-horn profile with peak SNR of 6.5 and linewidth at $50 \%$ of $160 \mathrm{~km} \mathrm{~s}^{-1}$. We recover a total $\mathrm{H}$ I flux of $0.7 \mathrm{Jy} \mathrm{km} \mathrm{s}^{-1}$ which at our distance of $118 \mathrm{Mpc}$ gives an $\mathrm{H} \mathrm{I}$ mass of $2.3 \times 10^{9} \mathrm{M}_{\odot}$. A search of the SDSS field revealed no other objects within a \pm 300 $\mathrm{km} \mathrm{s}^{-1}$ velocity range.

Assuming that the H I profile is unconfused, the asymmetric line shape and offset from the optical velocity is suggestive of bulk outflow of $\mathrm{H}$ I. Consistent with this interpretation is the observation in Paper II that LARS 01 shows extended $\mathrm{H} \alpha$ and $\mathrm{H} \beta$ emission. Further, preliminary results from COS spectroscopy show that within the COS aperture $\left(2.5^{\prime \prime}\right.$ diameter), large column densities of neutral hydrogen are outflowing at around $-128 \mathrm{~km} \mathrm{~s}^{-1}$ with respect to the $\mathrm{H} \alpha$ velocity. Note that LARS 01 will be studied in detail within Paper I, while Rivera-Thorsen et al. (in preparation) will present the COS analysis of the whole sample. Interstellar metal lines are blueshifted with respect to the systemic velocity of the galaxy, and there is a distinct lack of static gas in the COS aperture. This is consistent with the recent results from Wofford et al. (2013), whose analysis of the interstellar OI, Si II and CII lines in this galaxy also yields an outflow of the neutral gas at $-130 \mathrm{~km} \mathrm{~s}^{-1}$ (not including the geocoronal velocity offset applied by these authors). This large shift is also seen in our measurement of the systemic velocity of H I in LARS 01.

As the closest LARS galaxy, future interferometric H I observations of LARS 01 will be especially interesting. 


\subsection{LARS 02}

This galaxy is the strongest Ly $\alpha$ emitter (in terms of escape fraction) in the sample with a Ly $\alpha \mathrm{EW} \sim 82 \AA$, yet has one of the lowest (H $\alpha$ or UV-based) star formation rates. The GBT spectrum is double peaked (see Figure 4) and has a linewidth $\sim 140 \mathrm{~km} \mathrm{~s}^{-1}$. The total single-dish $\mathrm{H}$ I flux is $0.7 \mathrm{Jy} \mathrm{km} \mathrm{s}^{-1}$, which gives a mass of $2.7 \times 10^{9} \mathrm{M}_{\odot}$ at our derived distance of $127 \mathrm{Mpc}$. We find no sources of potential contamination in the SDSS field.

The VLA images of LARS 02 (see Figure 7) reveal a compact distribution of H I gas; the $\mathrm{H} \mathrm{I}$ is only slightly resolved by the $59^{\prime \prime}(36.3 \mathrm{kpc})$ beam. The H I column densities peak at $\sim 2 \times 10^{20} \mathrm{~cm}^{-2}$ (although the marginally resolved nature of the source strongly suggests that the $\mathrm{H}$ I distribution is more localized, with correspondingly higher mass surface densities). There is evidence for coherent rotation in this source; the isovelocity contours shown in panel (b) of Figure 7 are parallel from $\sim-15-15 \mathrm{~km} \mathrm{~s}^{-1}$ of the systemic velocity of $8960 \mathrm{~km} \mathrm{~s}^{-1}$. A coarse estimate of the dynamical mass of the system can be made by assuming that this rotational velocity is occurring over the angular diameter of the beam, and that the total projected rotation velocity is symmetric about a dynamical center. The resulting $\mathrm{M}_{\mathrm{dyn}}=$ $3.8 \times 10^{9} \mathrm{M}_{\odot}$ (which will increase for any non-zero value of disk inclination, but will decrease depending on the actual size of the rotating component of the disk) is consistent with the interpretation in Paper II of LARS 02 being among the more dwarf-like systems in LARS. We stress that this dynamical mass estimate is meant to be representative only.

\subsection{LARS 03}

LARS 03 is the nuclear region of the southeastern galaxy in the spectacular Arp 238 interacting pair. Note that Figure 1 shows the large-scale interacting morphology very clearly in the SDSS panel, while the HST Ly $\alpha$ imaging covers only the southern of the two nuclei, and only a relatively small portion of the total interacting system. Paper II finds that LARS 03 is a weak Ly $\alpha$ emitter. Interestingly, the Ly $\alpha$ luminosity increases as a function of distance from the source, suggesting that Ly $\alpha$ photons are readily being scattered to large distances even though the Ly $\alpha$ extension parameter $\left(\xi_{L y \alpha}\right.$, defined as the ratio between the Ly $\alpha$ and $\mathrm{H} \alpha$ Petrosian radii) is unity.

Based on the interacting morphology of the system, we expect both an irregular global H I profile and extended H I structure. Both of these expectations are borne out by the data. The GBT profile of LARS 03 (see Figure 4) is double-peaked and asymmetric. The linewidth exceeds $300 \mathrm{~km} \mathrm{~s}^{-1}$ at the $50 \%$ level, and there is evidence for low surface brightness $\mathrm{H} \mathrm{I}$ emission on even larger velocity scales $\left(\mathrm{W}_{20}=380 \pm 62 \mathrm{~km} \mathrm{~s}^{-1}\right.$, and the profile shows weak 
evidence for $\mathrm{H}$ I emission over a range as large as $\sim 600 \mathrm{~km} \mathrm{~s}^{-1}$ ).

The H I morphology and kinematics of LARS 03 clearly indicate a violent interaction between the two galaxies. The H I emission is extended many beam sizes beyond the optical galaxy. As shown in Hayes et al. (2013), Ly $\alpha$ emission is found in the southeast of the two interacting galaxies (although those authors point out that the nature of Ly $\alpha$ emission on larger spatial scales is not constrained by data presently in hand). Our VLA images reveal that the H I surface density maximum is coincident with the Northwest of the two interacting galaxies (although this is within one beam width at the present spatial resolution). This region is not included in the present HST observations. The total H I mass in the interacting system is $\sim 9 \times 10^{9} \mathrm{M}_{\odot}$, and the average $\mathrm{H}$ I column density as revealed by our VLA beam is $4.8 \times 10^{19} \mathrm{~cm}^{-2}$. An enormous tidal feature, which is contiguous in velocity space from the two interacting galaxies, extends $\sim 160 \mathrm{kpc}$ to the west-southwest and contains $\sim 1.6 \times 10^{9}$ $\mathrm{M}_{\odot}$ of HI gas. Because of the large distance separating this component from the main body of LARS 03 we decide to remove this mass from the total H I mass estimate of LARS 03 itself. This extended tidal structure is reminiscent of that in the local Ly $\alpha$-emitting starburst galaxies Tol 1924-416 and IRAS 08339+6517 found by Cannon et al. (2004), in which the large-scale neutral gas kinematics are interpreted to be critical for Ly $\alpha$ radiative transport. Although we do not probe the same physical scales in this work, we can conclude from this large tidal feature that the neutral gas is strongly disturbed.

Even with these interesting $\mathrm{H}$ I kinematics and the obvious interacting nature of the system, which contributes to its luminous infrared nature, LARS 03 remains a relatively weak emitter of Ly $\alpha$ photons, that would be undetected in most high-redshift surveys. While the ever increasing Ly $\alpha$ emission to higher radii is tantalizing, more data is needed to draw firm conclusions.

\subsection{LARS 04}

LARS 04 has a strong single peaked H I profile with a width at $50 \%$ of $150 \mathrm{~km} \mathrm{~s}^{-1}$ (see Figure 4). We classify it as an unconfused source; although SDSS J130757.13+542310.6 (V opt $=9714 \mathrm{~km} \mathrm{~s}^{-1}$ ) is located $5.56^{\prime}$ to the southwest, it is not detected in our VLA imaging (see below). LARS04 has an irregular morphology in the UV and is a net Lya absorber, just showing a weak asymmetric emission on top of a large, damped absorption profile within the HST COS aperture (2.5" in diameter). Paper II has shown that weak diffuse Ly $\alpha$ emission is present, but scattered to very large galactocentric radii.

We recover a total single-dish $\mathrm{H}$ I flux of $1.6 \mathrm{Jy} \mathrm{km} \mathrm{s}^{-1}$ which yields an $\mathrm{H}$ I mass of 
$7.3 \times 10^{9} \mathrm{M}_{\odot}$ at the adopted distance of $140 \mathrm{Mpc}$. The VLA H I imaging (see Figure 9) reveals extended emission with a median H I column density of $4.1 \times 10^{19} \mathrm{~cm}^{-2}$ [maximum angular extent roughly twice that of the $71^{\prime \prime}(47.5 \mathrm{kpc})$ beam]; interestingly, this extended $\mathrm{H}$ I component is in the same directional sense as the optical morphology. Higher spatial resolution observations of LARS 04 with the VLA are both technically feasible (sufficient source brightness) and will be very useful in assessing the localized morphology and kinematics.

There is coherent rotation of the source apparent at this low spatial resolution. Specifically, the isovelocity contours are mostly parallel over $\sim 100 \mathrm{~km} \mathrm{~s}^{-1}$ (see Figure 9). Using the same approach as for LARS 02 above, this implies a dynamical mass of $\mathrm{M}_{\mathrm{dyn}}=1.4 \times 10^{10} \mathrm{M}_{\odot}$ (again, with no inclination correction and with the assumption of the $\mathrm{H}$ I rotating over one beam diameter). However, based on the highly irregular optical morphology (which Figure 1 shows to be suggestive of an interaction), it is likely that the localized neutral gas kinematics are more complicated than simple disk rotation; the coarse beam size is likely smoothing out the kinematic details of this system.

\subsection{LARS 05}

LARS 05 is an edge-on galaxy (see Figure 1), with net Ly $\alpha$ absorption at very small radii and emission at larger radii, and at the physical scales relevant in this work [see discussion in Paper II]. The GBT H I profile (see Figure 4) shows a very weak signal that is near the noise level. While the SNR is low, the peaks in the spectrum may represent the top of the horns of a typical double-horn profile that is expected for a highly inclined disk galaxy. We derive an $\mathrm{H} \mathrm{I}$ flux integral of $0.55 \mathrm{Jy} \mathrm{km} \mathrm{s}^{-1}$, which, at a distance of $140 \mathrm{Mpc}$, corresponds to an H I mass of $2.4 \times 10^{9} \mathrm{M}_{\odot}$. This galaxy has no known companions or contaminants in the GBT field of view.

\subsection{LARS 06}

LARS 06 is an irregular galaxy that has the weakest $\mathrm{H} \alpha$ and UV-based star formation rates in the entire LARS sample; it is a $\operatorname{Ly} \alpha$ absorber on all physical scales. The optical morphology suggests an interaction scenario, with the southern tail being UV luminous but $\mathrm{H} \alpha$ dim compared to the main star forming knots in the northern portion of the system. Interestingly, this is the LARS system with the lowest measured nebular abundance [see discussion in Paper II].

The GBT H I profile of the LARS 06 field has the highest SNR of any of the LARS 
galaxies. This is almost certainly due to confusion within the beam. As Figure 5 shows, LARS 06 is separated from the disk galaxy UGC 10028 by only $\sim 1^{\prime}$ (43 kpc at the adopted distance of $148 \mathrm{Mpc})$. Although the redshift-derived velocity values differ for these two

galaxies by $\sim 200 \mathrm{~km} \mathrm{~s}^{-1}$, the $\mathrm{H}$ I velocity of the total system is centered directly at the velocity of UGC 10028. This single-dish H I spectrum is a classical double horn profile indicative of the rotation of a massive and inclined $\mathrm{H}$ I disk; the width of the profile is $370 \mathrm{~km} \mathrm{~s}^{-1}$ at $50 \%$ of the maximum. The $\mathrm{H} \mathrm{I}$ flux of $4.4 \mathrm{Jy} \mathrm{km} \mathrm{s}^{-1}$ yields a total $\mathrm{H} \mathrm{I}$ mass of $2.3 \times 10^{10} \mathrm{M}_{\odot}$; this can be compared with the estimate of the stellar mass of LARS 06, $2.1 \times 10^{9} \mathrm{M}_{\odot}$, from Paper II. While gas to stellar mass ratios of this size are not unreasonable, a comparison of the optical morphologies of UGC 10028 and LARS 06 strongly suggests that the former system is contributing significantly to the flux in this field. From the SDSS gband luminosity, we estimate the H I mass of UGC 10028 as $4.1 \times 10^{9} \mathrm{M}_{\odot}$. This represents roughly $20 \%$ of the total mass seen in the system, but we caution that this might be an over or under estimation if the galaxy does not follow the same relation as other galaxies.

There are three other galaxies in the vicinity of LARS 06 that could also be contributing to the observed flux integral. As discussed in the caption of Figure 5, LARS 06 and UGC 10028 are also in close angular proximity to 2MASX J15455278+4415470, 2MASX J15455157+4415310, and SDSS J154549.41+441539.2. Based on the optical velocity, 2MASX J15455278+4415470 appears to be roughly $1000 \mathrm{~km} \mathrm{~s}^{-1}$ in the background of LARS 06 and UGC 10028. Only photometric redshifts are available for 2MASX J15455157+4415310 and SDSS J154549.41+441539.2, and each appears to also be at larger distances than LARS 06 and UGC 10028; emission line spectroscopy will be required to determine the absolute velocities of these systems.

Wide-bandwidth VLA observations of this complex field will be able to localize the neutral gas components of each system; this is an ideal target for subsequent high spatial resolution observations. LARS 06 is also the only detected galaxy that lies significantly off of our observed gas fraction-stellar mass relationship (see discussion in $\S 6$ below), most likely due to this contamination.

\subsection{LARS 07}

LARS 07 is a near edge-on disk system that shows one of the highest Ly $\alpha$ equivalent widths of the LARS sample. The GBT spectrum shown in Figure 4 is measured as a single peak profile with a linewidth of $\sim 92 \mathrm{~km} \mathrm{~s}^{-1}$ at $50 \%$ of maximum; a second peak is separated from this main component by $\sim 120 \mathrm{~km} \mathrm{~s}^{-1}$. Due to the low SNR of this putative second peak, we measure the H I properties of the source using only the higher SNR component. 
With this assumption, LARS 07 has the narrowest linewidth of any galaxy in our sample. The H I flux is $0.47 \mathrm{Jy} \mathrm{km} \mathrm{s}^{-1}$, corresponding to an $\mathrm{H} \mathrm{I}$ mass of $2.9 \times 10^{9} \mathrm{M}_{\odot}$ at the adopted distance of $161 \mathrm{Mpc}$.

\subsection{LARS 08}

LARS 08 is a metal-rich, low-inclination system (see Figure 2 and discussion in Paper II). The distribution of $\mathrm{UV}, \mathrm{Ly} \alpha$, and $\mathrm{H} \alpha$ emission is strongly asymmetric in the galaxy, being much more prominent on the western side of the disk than on the eastern side. The system is a Ly $\alpha$ emitter on all spatial scales.

The GBT spectrum of LARS 08 (see Figure 4) shows a broad, possibly double-peaked profile with a linewidth of $310 \mathrm{~km} \mathrm{~s}^{-1}$ at $50 \%$ of the peak. The single-dish $\mathrm{H}$ I integrated flux is $3.4 \mathrm{Jy} \mathrm{km} \mathrm{s}^{-1}$, yielding a total $\mathrm{H} \mathrm{I}$ mass of $2.2 \times 10^{10} \mathrm{M}_{\odot}$ at the adopted distance of $160 \mathrm{Mpc}$. The galaxy appears to be isolated, with no known neighbors in the GBT beam with similar velocities. The median column density of $\mathrm{H} \mathrm{I}$ is $2.5 \times 10^{19} \mathrm{~cm}^{-2}$.

As Figure 10 shows, the detected H I emission is highly localized to the $72^{\prime \prime}$ (56.9 $\mathrm{kpc}$ ) beam. The source appears to be undergoing bulk rotation; while the velocity field is formally unresolved, the nearly parallel isovelocity contours span $\sim 80 \mathrm{~km} \mathrm{~s}^{-1}$ (note that the first moment map represents intensity-weighted velocity; for faint, unresolved sources one would expect a compressed velocity scale in the first moment compared to a spectrum). Taken as projected rotation with no inclination correction (which is likely substantial, given the apparent optical inclination), the implied dynamical mass is $\sim 1.1 \times 10^{10} \mathrm{M}_{\odot}$. Higher spatial resolution H I observations of LARS 08 would be very interesting to pursue, since this system appears to be undergoing normal rotation and lacks obvious signatures of large-scale kinematic disturbances in our H I maps, even though COS spectroscopy shows significant outflow activity (see details in Rivera-Thorsen et al., in preparation).

\subsection{LARS 09}

LARS 09 is an extended system with two prominent arms that are rich in $\mathrm{H} \alpha$-emitting star formation regions (note that a careful inspection of Figure 2 shows that there is a prominent foreground star at the southern end of the disk). The optical morphology is consistent with the source being either a loose spiral or an interacting pair. The system is a global Ly $\alpha$ emitter at large scales $(\mathrm{R}>10 \mathrm{kpc})$; the Ly $\alpha$ morphology is similar to that of the optical/UV, although more extended in all directions from the disk. 
The GBT spectrum (see Figure 4) is broad (linewidth of $270 \mathrm{~km} \mathrm{~s}^{-1}$ at $50 \%$ of the peak) and relatively low SNR. The total $\mathrm{H}$ I single-dish flux is $1.2 \mathrm{Jy} \mathrm{km} \mathrm{s}^{-1}$, which gives a mass of $1.2 \times 10^{10} \mathrm{M}_{\odot}$ at a distance of $200 \mathrm{Mpc}$. The profile is not contaminated by any other known sources within the GBT beam. The median H I column density is $1.2 \times 10^{19} \mathrm{~cm}^{-2}$

The VLA images presented in Figure 11 show that the source is essentially unresolved by the $59^{\prime \prime}(57.2 \mathrm{kpc})$ beam. The H I maximum is co-spatial with the optical body; there is weak evidence for extended gas that is only slightly larger than the beam size (see, for example, the extended material to the east-southeast of the optical body in Figure 11). However, we do not interpret this as evidence of extended tidal structure due to the marginal extension compared to the beam size.

The intensity weighted velocity field of LARS 09 covers a large range $\left(>200 \mathrm{~km} \mathrm{~s}^{-1}\right)$ and is severely disturbed. There are some isovelocity contours that are roughly parallel in the northwest region of the system; however, at the position of the optical body these contours deviate significantly from regularity. The kinematic information in these images suggests that the interaction scenario for the optical morphology may be appropriate. The presence of diffuse Ly $\alpha$ emission in this source, combined with the irregular kinematics and morphology, make this a prime target for higher resolution H I observations.

\subsection{LARS 10}

LARS 10 is an irregular source that shows high Ly $\alpha$ equivalent width emission arising from a diffuse component that extends beyond the optical body of the source [see discussion in Paper I]. The optical morphology is consistent with an advanced merger state. The GBT profile is broad ( $\sim 280 \mathrm{~km} \mathrm{~s}^{-1}$ at $50 \%$ of maximum), although of low SNR; the total flux integral of $0.29 \mathrm{Jy} \mathrm{km} \mathrm{s}^{-1}$ corresponds to a total $\mathrm{H}$ I mass of $\sim 4.1 \times 10^{9} \mathrm{M}_{\odot}$ at the adopted distance of $250 \mathrm{Mpc}$. The weak H I flux integral will make high resolution interferometric observations of LARS 10 challenging.

\subsection{LARS 11}

LARS 11 is a dramatic edge-on galaxy that is in close angular proximity to a field spiral galaxy (CGCG 046-044 NED01 at $14^{\mathrm{h}} 03^{\mathrm{m}} 47.0^{\mathrm{s}},+06^{\circ} 28^{\prime} 26^{\prime \prime}$ ). LARS 11 appears to be in the foreground of this object (LARS 11 is at $\mathrm{z}_{\mathrm{opt}}=0.0843$, while CGCG 046-044 NED01 has a photometric redshift derived from SDSS of $\sim 0.1$ ); this is apparent by the disk of LARS 11 visually obstructing one of the spiral arms of CGCG 046-044 NED01. Figure 6 shows that 
LARS 11 is separated from two other field galaxies with similar velocities by less than the GBT beam: SDSS J140401.00+062901.7 at $14^{\mathrm{h}} 04^{\mathrm{m}} 01.0^{\mathrm{s}}+06^{\circ} 29^{\prime} 02^{\prime \prime}$, and SDSS J140353.36+062504.8 at $14^{\mathrm{h}} 03^{\mathrm{m}} 53.3^{\mathrm{s}}+06^{\circ} 25^{\prime} 05^{\prime \prime}$. Given this complex field, we classify the GBT profile of LARS 11 as potentially confused. Further, RFI was present near the expected frequency of the H I spectral line from LARS 11; this required extensive flagging.

The H I profile of LARS 11 shown in Figure 4 is broad $\left(\mathrm{W}_{50}=260 \mathrm{~km} \mathrm{~s}^{-1}\right)$ and is distributed in multiple peaks. This may represent a broad rotation profile from LARS 11, or it may be indicative of multiple sources contributing to the H I flux within the beam. Assuming that the full $\mathrm{H}$ I flux integral of $0.75 \mathrm{Jy} \mathrm{km} \mathrm{s}^{-1}$ is only associated with LARS 11, the implied neutral hydrogen mass is $2.3 \times 10^{10} \mathrm{M}_{\odot}$ at the adopted distance of $360 \mathrm{Mpc}$. VLA imaging of LARS 11 would be very useful for localizing the $\mathrm{H}$ I emission from the various sources in this complex field.

\section{Global Characteristics of the LARS Galaxies}

Due to resonant scattering with neutral hydrogen atoms, the radiative transport of Ly $\alpha$ photons may well have its strongest dependence on the characteristics of the $\mathrm{H}$ I that surrounds the star forming regions in which the photons are produced. With the present H I spectroscopy and imaging, we now have a first order understanding of the neutral gas contents of many of the LARS galaxies. Comparing the qualities of the global H I reservoirs with the detailed characteristics of each system derived from HST imaging allows us to probe what roles H I kinematics and density play in governing Ly $\alpha$ radiative transport.

We focus on four global properties derived from the present H I observations of the 11 lowest-redshift LARS galaxies (those for which the GBT spectra provide meaningful measurements or limits); for the galaxies where it is available (LARS 02,03,04,08,09) we use the total fluxes and masses VLA images preferentially over the GBT data. In general the VLA data recovers $30 \%$ less flux than the GBT data, which contributes to $6 \%$ shorter linewidths. As mentioned in section 4, this is most likely due to a combination of decreased surface brightness sensitivity in the VLA data and decreased contamination from other sources.

First, the H I linewidth (specifically, the width at $50 \%$ of the maximum $\mathrm{W}_{50}$ ) is a distance-independent variable that has been shown to correlate with absolute magnitude and thus to serve as a proxy for mass in star-forming disk galaxies (Tully \& Fisher 1977). For rotationally dominated galaxies, a larger H I linewidth can often be interpreted as an indicator of a more massive galaxy. To know with certainty, a detailed study of the dynamics 
and complete censuses of the baryonic components of individual galaxies is needed. In our case, the galaxies with irregular morphology may have linewidths that are significantly increased due to kinematic disturbances and merging activity.

The second global parameter we examine is the total H I mass of each LARS galaxy. This parameter is of course related to the H I linewidth, but not in a one to one sense. The LARS galaxies span a range of morphological types, including violent interactions (e.g., LARS 03), disk-dominated spirals (e.g., LARS 05 and LARS 11), and irregulars (e.g., LARS 04, LARS 06). While for disk dominated systems the H I linewidth and H I mass should be closely correlated, for the other types of systems the H I line profile may not only be indicative of rotation; H I gas from multiple components may create irregular single-dish profiles. Examples of this are clearly seen in the GBT spectra (Figure 4) and in the VLA images (see, e.g., Figure 8).

Third, we examine the offset in velocity between the centroid of the $\mathrm{H}$ I profile and the known systemic velocity of each LARS galaxy as derived from optical spectroscopy. Assuming that such offsets are caused by large-scale kinematic deviations from regularly rotating galaxy disks (e.g., tidal interaction, gas outflow or infall), this offset parameter can be considered a rough proxy for the presence or absence of large-scale motions of neutral gas. As discussed in Cannon et al. (2004) and above for LARS 03, we find evidence for extended tidal structure in some Ly $\alpha$-emitting galaxies. However, we also find systems that are strong Ly $\alpha$ emitters that do not show such extended neutral gas components (e.g., LARS 02; see $\S 5.2$ above).

Finally, we examine the gas mass fraction defined as the H I mass divided by the stellar mass. Numerous works have shown that normal, star-forming disk galaxies populate a robust trend of decreasing gas fraction with increasing stellar mass (see recent results in Huang et al. 2012, Papastergis et al. 2012, Peeples et al. 2014, and various references therein). In Figure 12 we show the gas fraction as a function of total stellar mass $\left(\mathrm{M}_{*}\right)$ derived from 2-component SED modeling to the HST data (see Paper II and Hayes et al. 2009 for details). Except for LARS 06, whose H I flux integral is likely strongly contaminated by the nearby field spiral UGC 10028 (see discussion in $\S 5.6$ and Figure 5), the LARS galaxies closely follow the derived relations for nearby galaxies as found in Papastergis et al. (2012) and Peeples et al. (2014).

It is important to remain cognizant of the possible confusion within the GBT beam for some of the sources without VLA data. In particular, the H I properties of LARS 06 are almost certainly contaminated by UGC 10028, and those of LARS 11 may be contaminated by SDSS J140401.00+062901.7 and/or SDSS J140353.36+062504.8. If these sources are in fact contaminated, then the $\mathrm{H}$ I linewidths and masses will be overestimated; the velocity 
offsets may or may not change.

We examine the relationships of these four H I-based quantities with seven global properties derived from HST data in Paper II: the $\mathrm{Ly} \alpha / \mathrm{H} \alpha$ flux ratio, the Ly $\alpha$ luminosity, the $\mathrm{H} \alpha / \mathrm{H} \beta$ flux ratio, the Ly $\alpha$ escape fraction, the Ly $\alpha$ equivalent width, the Ly $\alpha$ extension parameter $(\operatorname{Ly} \alpha \xi)$, and the SFR per unit area within the Petrosian radius $(\eta=0.2$; see Paper II). As discussed in detail in Hayes et al. (2014), there is a complex interrelation between these (and other) properties in the process of Ly $\alpha$ radiative transport; the total Ly $\alpha$ luminosity of a given galaxy does not correlate in a statistically significant way with any individual quantity. Further, there is significant variation in these values as a function of position within an individual galaxy; some galaxies appear as net absorbers within the disk and as net emitters when the diffuse Ly $\alpha$ halo is included. Nevertheless, we seek statistical comparisons with many of the global quantities of both Ly $\alpha$ and H I. These comparisons most closely match detections of higher-redshift Ly $\alpha$ emitters and will constrain one of the many poorly understood aspects of Ly $\alpha$ propagation and detection in the distant universe.

From a simplified interpretative standpoint, these seven global quantities can be related as follows. The $\mathrm{H} \alpha / \mathrm{H} \beta$ ratio indicates extinction; deviations above the intrinsic ratio of 2.86 Osterbrock 1989) indicate non-zero $\mathrm{E}(\mathrm{B}-\mathrm{V})$ values. These $\mathrm{E}(\mathrm{B}-\mathrm{V})$ values can in turn be used to derive the intrinsic Ly $\alpha$ luminosity of a system based on its observed (and extinction corrected) $\mathrm{H} \alpha$ luminosity and also to estimate the Ly $\alpha$ escape fraction. The $\mathrm{Ly} \alpha / \mathrm{H} \alpha$ ratio has a (Case B) recombination value of 8.7. Lower values can indicate stronger suppression of Ly $\alpha$ versus $\mathrm{H} \alpha$ (e.g., by attenuation from dust, or by resonant scattering away from the production site); super-recombination values are seen in a few localized regions of the LARS galaxies, as well as in some of the large scattered Ly $\alpha$ halos surrounding some of the systems (see Paper I and Paper II). The Ly $\alpha$ equivalent width is related to the recent star formation history of the galaxy or region; global values of $\sim 100 \AA$ indicate constant star formation over $\sim 100$ Myr timescales, while values exceeding $250 \AA$ occur only in the very youngest burst episodes (Schaerer et al. 2003 and Raiter et al. 2010.)

We present scatter plot comparisons of the four global H I-derived properties and seven global HST-derived properties in Figures 13, 14, 16, and 15. We restrict the Ly $\alpha$ properties to positive values, which has the effect of setting EW and luminosity measurements equal to zero. We mark this region on the plots with a hashed region. We use the Spearman $\rho_{s}$ correlation coefficient to quantify possible monotonic correlations between these properties; a perfect correlation or anti-correlation between two properties will have $\rho_{s}$ values of +1 or -1 , respectively. Each panel of Figures 13 through 16 shows the corresponding $\rho_{s}$ value. To estimate the errors on these correlation coefficients we resample the properties 1000 times with a random realization of the associated errors and measure, recording the correlation 
coefficients each time. For each correlation we show the value from the sample of uncontaminated detections as the primary result, and include the value for the entire sample including upper limits and confused detections below.

Overall the results do not show strong evidence of correlation between properties $\left(\rho_{s}<\right.$ -0.6 or $\left.\rho_{s}>+0.6\right)$. We present all correlations in Table 6, along with the dispersion and discuss the correlations for each H I property below.

\subsection{Linewidth}

The H I line width is significantly anti-correlated with the Ly $\alpha$ extension parameter,

$\xi_{\text {Ly } \alpha}$ (see figure 14). This is the strongest evidence of correlation in the entire sample. The $\mathrm{H}$ I line width is positively correlated with the $\mathrm{H}_{\alpha} / \mathrm{H}_{\beta}$ ratio (see further discussion below). Two factors are possibly at play here. Either larger H I quantities scatter Ly $\alpha$ photons to extended radii, or interactions increase both the linewidth and preferentially block Ly $\alpha$ photons at short radii.

\subsection{Mass}

Two anti-correlations, and a positive correlation appear comparing the H I mass of our sample of well detected galaxies (see Figure 13). The escape fraction $\left(\rho_{s}=-0.63\right)$, and Ly $\alpha$ EW $\left(\rho_{s}=-0.60\right)$ show tentative evidence for anti-correlation, which do not appear when including the marginal detections. Interestingly, the $\mathrm{H}_{\alpha} / \mathrm{H}_{\beta}$ ratio shows strong evidence of correlation $\left(\rho_{s}=0.75\right)$. It has been known for some time that more massive star forming galaxies (hence those with larger H I masses and linewidths) are dustier, and that the Balmer decrement scales accordingly (e.g., Brinchmann et al. 2004; Lee et al. 2009; Garn \& Best 2010). How this correlation may be connected to the underling Ly $\alpha$ propagation is not entirely clear.

\subsection{Gas Fraction}

We see no conclusive evidence of correlations with any of the global Ly $\alpha$ properties using either the whole sample or the positive detections. The strongest correlation is with the $\mathrm{H} \alpha / \mathrm{H} \beta$ ratio $\left(\rho_{s}=-0.53\right)$. 


\subsection{Velocity Offset}

We find no significant correlation between the velocity offsets and the various Ly $\alpha$ properties. This might be due to our velocity smoothing and the large extent over which we are probing the velocity information. The strongest correlation is with with the $\mathrm{H} \alpha / \mathrm{H} \beta$ ratio $\left(\rho_{s}=0.50\right)$.

\section{Discussion and Conclusions}

We have presented new GBT spectroscopy and VLA H I spectral line imaging of the galaxies in the "Lyman Alpha Reference Sample" ("LARS"). LARS is a comprehensive, multi-wavelength study of $14 \mathrm{UV}$-selected star-forming galaxies that functions as a local benchmark for studies of Ly $\alpha$ emission and absorption at higher redshifts. The centerpiece is a suite of HST images that allows us to study Ly $\alpha$ emission and related quantities on a spatially resolved basis. The preliminary results presented in Hayes et al. (2013) showed that most of the LARS galaxies have large, diffuse halos of Ly $\alpha$ emission that exceed the sizes of the stellar populations; this is strong evidence for the importance of resonant scattering in the neutral hydrogen gas component. The HI structure and content are important pieces for understanding the origin of the Ly-alpha halos and their different sizes. Our current HI angular resolution probes the large-scale global properties rather than those in the immediate vicinity of the Ly-alpha halo regions imaged with HST. Nevertheless, these provide important insights into the galaxy structure and environment. The LARS survey products are described

in Paper I of this series (Östlin et al. 2014); the integrated properties of the LARS galaxies as derived from the HST imaging are presented in Paper II of this series (Hayes et al. 2014).

In this manuscript we present a first exploration of the H I properties of the LARS galaxies. Using data from the GBT $100 \mathrm{~m}$ telescope, we have presented direct measurements of the neutral gas contents of 11 of the 14 galaxies; we place limits on the three most distant $(\mathrm{z}>0.1)$ LARS galaxies. These profiles show a wide variety of structure. Some systems harbor multi-component profiles that are indicative of interaction or the presence of nearby companions; others show more simple single H I peaks. Two sources (LARS 06 and LARS 11) are likely confused with nearby galaxies. We fit each of these profiles in order to derive global line properties, including systemic velocity, linewidths, H I flux integrals, and H I masses.

For a subset of five of the LARS galaxies, we also present new, low-resolution H I spectral line imaging obtained with the VLA. These H I images allow us to localize the H I associated with each galaxy. Three of the systems (LARS 02, LARS 08, and LARS 09) are unresolved at this angular resolution, while LARS 04 is resolved by a few beams. LARS 03 
is highly resolved by the present data; we have discovered an enormous tidal structure that contains more than $10^{9} \mathrm{M}_{\odot}$ of $\mathrm{H} \mathrm{I}$, and that extends more than $160 \mathrm{kpc}$ from the main interacting galaxies in the LARS 03 system. Based on the recovered flux integrals from these VLA data, each of the five systems presented here would be amenable to higher spatial resolution observations. The VLA C and B configurations would offer a factor of roughly 4 and 10 improvement in resolution respectively, but at the cost of longer integration times.

In particular LARS 03 presents an interesting case where we have unequivocal evidence of kinematic disturbances on large scales, yet no accompanying boost relative to nondisturbed galaxies in Ly $\alpha$ escape fraction. A high resolution follow up with the VLA and future radio telescopes, especially coupled with larger maps of the Ly $\alpha$ flux, would disentangle the local versus global kinematic effects, and probe the Ly $\alpha$ escape on physical scales larger than the $\sim 10 \mathrm{kpc}$ already probed in the HST imaging.

Using these new GBT and VLA data, as well as the results derived from HST imaging and presented in Paper II, we compare various global H I and Ly $\alpha$ properties. While the sample is small, we find a few intriguing correlations in our robustly detected galaxies: The $\mathrm{H}$ I linewidths are strongly anti-correlated with $\xi_{\mathrm{Ly} \alpha}\left(\rho_{s}=-0.81\right)$, while the total $\mathrm{H} \mathrm{I}$ masses show anti-correlations with escape fraction and Ly $\alpha$ EW. Additionally, both the H I linewidths and the H I masses are each correlated with the $\mathrm{H}_{\alpha} / \mathrm{H}_{\beta}$ ratio. These intriguing but tentative (anti-)correlations are in general agreement with a significant dependence of Ly $\alpha$ propagation on the total mass and thus the $\mathrm{H}$ I mass of the source, and is in general agreement with previous work by Laursen et al. (2009) which found that the escape fraction decreases with increasing virial mass. These results also support the general trend that Ly $\alpha$ properties appear to correlate with total galaxy mass, as found in Paper II. That work showed that Ly $\alpha$ emission (quantified by Ly $\alpha$ equivalent width, escape fraction, etc.) is systematically larger in lower mass galaxies. Given the variety of properties that scale roughly with mass (e.g., metallicity, dust content, star formation rate, UV colors, etc.), a larger statistical sample of galaxies will be needed in order to better quantify these trends.

The famous metal-poor blue compact dwarf galaxy I Zw18 reminds us that the trend of lower mass galaxies having larger Ly $\alpha$ luminosities or escape fractions is not always true. While the system has an extremely low nebular oxygen abundance (Skillman \& Kennicutt 1993), an appreciable star formation rate (Cannon et al. 2002), and a substantial UV luminosity (Grimes et al. 2009), Kunth et al. (1998) showed that I Zw 18 is in fact a Ly $\alpha$ absorber. This process was explained by numerous scattering events in the neutral, and, most importantly, static ISM even in the complete absence of dust (Atek et al. 2009). Future local Ly $\alpha$ studies should seek to expand the number of low-mass systems with analyses similar to the ones presented here for LARS. 
Although it is tempting to view the increasing $\mathrm{Ly} \alpha / \mathrm{H} \alpha$ with decreasing mass as a result of less gas mass being available for scattering, this theory must be cast in the light of results that suggest that gas fraction increases with increasing redshift (e.g., Tacconi et al. 2013), and that lower mass galaxies tend to be more gas-rich (Saintonge et al. 2011) and less dusty (e.g., Blanc et al. 2011). In Figure 12 we see that the LARS galaxies fit the general trend with gas mass fraction.

While Ly $\alpha$ halos seem to appear in systems with a range of $\mathrm{H}$ I linewidths, large $\mathrm{Ly} \alpha / \mathrm{H}_{\alpha}$ values only appear in galaxies with $\mathrm{H}$ I smaller linewidths. Large Ly $\alpha$ halos are also correlated with less massive galaxies, and with increasing velocity offsets. We interpret this to mean that low H I linewidths are necessary but not sufficient for Ly $\alpha$ escape, while larger line widths contribute to the overall effects of Ly $\alpha$ destruction by dust, extinction, and scattering.

The bulk of the evidence presented by previous Ly $\alpha$ work shows the importance of $\mathrm{H} \mathrm{I}$ properties, especially kinematic details, in determining the escape of photons (e.g. MasHesse et al. 2003). This process is mostly governed by local effects, and it is unclear at this time what global H I properties are required for the escape and propagation of Ly $\alpha$ photons. The asymmetric GBT profiles and evidence of Ly $\alpha$ emission in the presence of large column densities of $\mathrm{H}$ I both point to interesting sub-resolution kinematic and density effects.

A larger sample of galaxies with data like those presented here will allow us to place these correlations on a more statistically robust footing. The HST eLARS program has been approved for 54 orbits and contains 28 more galaxies within our nominal sensitivity range of $\mathrm{z} \sim 0.08$. In addition, higher resolution follow up observations will allow us to determine kinematic details on more applicable scales for a number of our galaxies. This program will likely be the largest statistical sample of both H I and Ly $\alpha$ detected galaxies until new advances of radio telescopes, such as the Square Kilometer Array 7 , push H I detections to 20-100 kpc scales at $\mathrm{z}>1$.

The authors thank the anonymous referee for insightful comments that improved the quality of this manuscript. J.M.C. and S.P. thank Sung Kyu Kim and Macalester College for research support. J.M.C. would like to thank the Instituto Nazionale di Astrofisica and the Osservatorio Astronomico di Padova for their hospitality during a productive sabbatical leave. G.Ö. is a Royal Swedish Academy of Science research fellow (supported by a grant from the Knut \& Alice Wallenbergs foundation). M.H. acknowledges the support of the Swedish Research Council (Vetenskapsrådet) and the Swedish National Space Board (SBSB). This

\footnotetext{
${ }^{7}$ https://www.skatelescope.org
} 
work was supported by the Swedish Research Council and the Swedish National Space Board. H.O.F. is ended by a postdoctoral UNAM grant. J.M.M-H is funded by Spanish MINECO grants AYA2010-21887-C04-02 and AYA2012-39362-C02-01. I.O. was supported by the Sciex fellowship of the Rectors' Conference of Swiss Universities. P.L. acknowledges support from the ERC-StG grant EGGS-278202. D.K is supported by CNES (Centre National d'etudes spatiales) for the HST project.

This investigation has made use of the NASA/IPAC Extragalactic Database (NED) which is operated by the Jet Propulsion Laboratory, California Institute of Technology, under contract with the National Aeronautics and Space Administration, and NASA's Astrophysics Data System. This investigation has made use of data from the Sloan Digitized Sky Survey (SDSS). Funding for the SDSS and SDSS-II has been provided by the Alfred P. Sloan Foundation, the Participating Institutions, the National Science Foundation, the U.S. Department of Energy, the National Aeronautics and Space Administration, the Japanese Monbukagakusho, the Max Planck Society, and the Higher Education Funding Council for England. The SDSS Web Site is http://www.sdss.org/.

The SDSS is managed by the Astrophysical Research Consortium for the Participating Institutions. The Participating Institutions are the American Museum of Natural History, Astrophysical Institute Potsdam, University of Basel, University of Cambridge, Case Western Reserve University, University of Chicago, Drexel University, Fermilab, the Institute for Advanced Study, the Japan Participation Group, Johns Hopkins University, the Joint Institute for Nuclear Astrophysics, the Kavli Institute for Particle Astrophysics and Cosmology, the Korean Scientist Group, the Chinese Academy of Sciences (LAMOST), Los Alamos National Laboratory, the Max-Planck-Institute for Astronomy (MPIA), the Max-Planck-Institute for Astrophysics (MPA), New Mexico State University, Ohio State University, University of Pittsburgh, University of Portsmouth, Princeton University, the United States Naval Observatory, and the University of Washington. 


\section{REFERENCES}

Atek, H., Kunth, D., Hayes, M., Östlin, G., \& Mas-Hesse, J. M. 2008, A\&A, 488, 491

Atek, H., Kunth, D., Schaerer, D., et al. 2009, A\&A, 506, L1

Atek, H., Kunth, D., Schaerer, D., et al. 2014, A\&A, 561, A89

Bergvall, N., \& Jorsater, S. 1988, Nature, 331, 589

Blanc, G. A., Adams, J. J., Gebhardt, K., et al. 2011, ApJ, 736, 31

Brinchmann, J., Charlot, S., White, S. D. M., et al. 2004, MNRAS, 351, 1151

Cannon, J. M., Skillman, E. D., Garnett, D. R., \& Dufour, R. J. 2002, ApJ, 565, 931

Cannon, J. M., Skillman, E. D., Kunth, D., Leitherer, C., Mas-Hesse, M., Östlin, G., \& Petrosian, A. 2004, ApJ, 608, 768

Charlot, S., \& Fall, S. M. 1993, ApJ, 415, 580

Duval, F., Schaerer, D., Östlin, G., \& Laursen, P. 2014, A\&A, 562, A52

Finkelstein, S. L., Rhoads, J. E., Malhotra, S., Grogin, N., \& Wang, J. 2008, ApJ, 678, 655

Garn, T., \& Best, P. N. 2010, MNRAS, 409, 421

Giavalisco, M., Koratkar, A., \& Calzetti, D. 1996, ApJ, 466, 831

Grimes, J. P., Heckman, T., Aloisi, A., et al. 2009, ApJS, 181, 272

Hansen, M., \& Oh, S. P. 2006, MNRAS, 367, 979

Hartmann, L. W., Huchra, J. P., Geller, M. J., O’Brien, P., \& Wilson, R. 1988, ApJ, 326, 101

Hayes, M., Östlin, G., Mas-Hesse, J. M., et al. 2005, A\&A, 438, 71

Hayes, M., Östlin, G., Mas-Hesse, J. M., \& Kunth, D. 2009, AJ, 138, 911

Hayes, M., Östlin, G., Schaerer, D., et al. 2010, Nature, 464, 562

Hayes, M., Östlin, G., Schaerer, D., et al. 2013, ApJ, 765, L27

Hayes, M., Östlin, G., Duval, F., et al. 2014, ApJ, 782, 6

Haynes, M. P., \& Giovanelli, R. 1984, AJ, 89, 758 
Huang, S., Haynes, M. P., Giovanelli, R., et al. 2012, AJ, 143, 133

Jester, S., et al. 2005, AJ, 130, 873

Komatsu, E., et al. 2011, ApJS, 192, 18

Kunth, D., Mas-Hesse, J. M., Terlevich, E., et al. 1998, A\&A, 334, 11

Laursen, P., Sommer-Larsen, J., \& Andersen, A. C. 2009, ApJ, 704, 1640

Laursen, P., Duval, F., Östlin, G. 2013, ApJ, 766, 124

Lee, J. C., Gil de Paz, A., Tremonti, C., et al. 2009, ApJ, 706, 599

Mas-Hesse, J. M., Kunth, D., Tenorio-Tagle, G., et al. 2003, ApJ, 598, 858

Neufeld, D. A. 1991, ApJ, 370, L85

Osterbrock, D. E. 1989, Research supported by the University of California, John Simon Guggenheim Memorial Foundation, University of Minnesota, et al. Mill Valley, CA, University Science Books, 1989, 422 p.,

Östlin, G., Hayes, M., Kunth, D., et al. 2009, AJ, 138, 923

Östlin, G.,Hayes, M., et al. 2014, ApJ, in preparation

Otí-Floranes, H., Mas-Hesse, J. M., Jiménez-Bailón, E., et al. 2012, A\&A, 546, A65

Papastergis, E., Cattaneo, A., Huang, S., Giovanelli, R., \& Haynes, M. P. 2012, ApJ, 759, 138

Peeples, M. S., Werk, J. K., Tumlinson, J., et al. 2014, ApJ, 786, 54

Petrosian, V. 1976, ApJ, 209, L1

Raiter, A., Schaerer, D., \& Fosbury, R. A. E. 2010, A\&A, 523, A64

Saintonge, A., Kauffmann, G., Kramer, C., et al. 2011, MNRAS, 415, 32

Scarlata, C., Colbert, J., Teplitz, H. I., et al. 2009, ApJ, 704, L98

Schaerer, D. 2003, A\&A, 397, 527

Schlafly, E. F., \& Finkbeiner, D. P. 2011, ApJ, 737, 103

Schneider, S. E., Thuan, T. X., Magri, C., \& Wadiak, J. E. 1990, ApJS, 72, 245 
Skillman, E. D., \& Kennicutt, R. C., Jr. 1993, ApJ, 411, 655

Springob, C. M., Haynes, M. P., Giovanelli, R., \& Kent, B. R. 2005, ApJS, 160, 149

Stark, D. P., Ellis, R. S., Chiu, K., Ouchi, M., \& Bunker, A. 2010, MNRAS, 408, 1628

Steidel, C. C., Bogosavljević, M., Shapley, A. E., et al. 2011, ApJ, 736, 160

Tacconi, L. J., Neri, R., Genzel, R., et al. 2013, ApJ, 768, 74

Tully, R. B., \& Fisher, J. R. 1977, A\&A, 54, 661

Verhamme, A., Schaerer, D., Atek, H., \& Tapken, C. 2008, A\&A, 491, 89

Westmeier, T., Jurek, R., Obreschkow, D., Koribalski, B. S., \& Staveley-Smith, L. 2014, MNRAS, 438, 1176

Wofford, A., Leitherer, C., \& Salzer, J. 2013, ApJ, 765, 118

Zheng, Z., Cen, R., Weinberg, D., Trac, H., \& Miralda-Escudé, J. 2011, ApJ, 739, 62 
Table 1. Basic Properties of the LARS Sample

\begin{tabular}{|c|c|c|c|c|c|c|c|}
\hline Galaxy & $\begin{array}{l}\text { Alternate } \\
\text { name }\end{array}$ & $\begin{array}{c}\text { RA } \\
(\mathrm{J} 2000)\end{array}$ & $\begin{array}{c}\text { Dec } \\
(\mathrm{J} 2000)\end{array}$ & $\begin{array}{c}\mathrm{V}_{\mathrm{opt}^{\mathrm{a}}} \\
\left(\mathrm{km} \mathrm{s}^{-1}\right)\end{array}$ & $\mathrm{z}_{\mathrm{opt}}{ }^{\mathrm{a}}$ & $\begin{array}{c}\text { Distance }^{\mathrm{b}} \\
(\mathrm{Mpc})\end{array}$ & Morphological Type ${ }^{\mathrm{c}}$ \\
\hline LARS 01 & Mrk 259 & $13: 28: 44.50$ & $+43: 55: 50$ & 8394 & 0.028 & 120 & Dwarf Irregular \\
\hline LARS 02 & SDSS J090704.88+532656.6 & 09:07:04.88 & $+53: 26: 56$ & 8934 & 0.030 & 130 & Dwarf Irregular \\
\hline LARS 03 & Arp 238 & $13: 15: 35.60$ & $+62: 07: 28$ & 9204 & 0.031 & 130 & Merger \\
\hline LARS 04 & SDSS J130728.45+542652.3 & $13: 07: 28.45$ & $+54: 26: 52$ & 9743 & 0.033 & 140 & Irregular \\
\hline LARS 05 & Mrk 1486 & $13: 59: 50.91$ & $+57: 26: 22$ & 10133 & 0.034 & 150 & Edge-on Dwarf Spiral \\
\hline LARS 06 & KISSR 2019 & $15: 45: 44.52$ & $+44: 15: 51$ & 10223 & 0.034 & 150 & Dwarf Irregular \\
\hline LARS 07 & IRAS $1313+2938$ & $13: 16: 03.91$ & $+29: 22: 54$ & 11332 & 0.038 & 170 & Dwarf Edge-on Spiral \\
\hline LARS 08 & SDSS J125013.50+073441.5 & $12: 50: 13.50$ & $+07: 34: 41$ & 11452 & 0.038 & 170 & Spiral \\
\hline LARS 09 & IRAS $0820+2816$ & $08: 23: 54.96$ & $+28: 06: 21$ & 14150 & 0.047 & 210 & Edge-on Spiral \\
\hline LARS 10 & MRk 0061 & $13: 01: 41.52$ & $+29: 22: 52$ & 17208 & 0.057 & 260 & Merger \\
\hline LARS 11 & SDSS J140347.22+062812.1 & $14: 03: 47.22$ & $+06: 28: 12$ & 25302 & 0.084 & 380 & Edge-on Spiral \\
\hline LARS 12 & SBS $0934+547$ & $09: 38: 13.49$ & $+54: 28: 25$ & 30608 & 0.102 & 470 & Dwarf Irregular \\
\hline LARS 13 & IRAS $0147+1254$ & $01: 50: 28.39$ & $+13: 08: 58$ & 43979 & 0.147 & 700 & Irregular \\
\hline LARS 14 & SDSS J092600.40+442736.1 & 09:26:00.40 & $+44: 27: 36$ & 54172 & 0.181 & 880 & Dwarf \\
\hline
\end{tabular}

${ }^{\text {a }}$ Derived from SDSS spectroscopy.

${ }^{\mathrm{b}}$ Values derived from luminosity distance.

${ }^{\mathrm{c}}$ Morphologies provided to guide the reader and are presented from optical imaging and Paper II. Irregulars have no obvious spiral structure in the optical disk and mergers have an obvious interacting companion. Galaxies marked as dwarves have stellar masses lower than $10^{10} \mathrm{M}_{\odot}$. 
Table 2. Observed and Derived GBT H I Properties

\begin{tabular}{|c|c|c|c|c|c|c|c|c|c|c|}
\hline $\begin{array}{c}\text { Galaxy } \\
\text { (1) }\end{array}$ & $\begin{array}{c}\mathrm{V}_{\text {sys }^{\mathrm{a}}} \\
\left(\mathrm{km} \mathrm{s}^{-1}\right) \\
(2)\end{array}$ & $\begin{array}{c}\mathrm{W}_{50} \mathrm{~b}, c \\
\left(\mathrm{~km} \mathrm{~s}^{-1}\right) \\
(3)\end{array}$ & $\begin{array}{c}\mathrm{W}_{20}^{\mathrm{b}} \\
\left(\mathrm{km} \mathrm{s}^{-1}\right) \\
(4)\end{array}$ & $\begin{array}{c}\text { Velocity Offset } \\
\left(\mathrm{km} \mathrm{s}^{-1}\right) \\
(5)\end{array}$ & $\begin{array}{c}\mathrm{S}_{\mathrm{HI}}{ }^{\mathrm{d}} \\
\left(\mathrm{Jy} \mathrm{km} \mathrm{s}^{-1}\right) \\
(6)\end{array}$ & $\begin{array}{c}\mathrm{M}_{\mathrm{HI}} \\
\left(10^{9} \mathrm{M}_{\odot}\right) \\
(7)\end{array}$ & $\begin{array}{c}\text { SNR } \\
(\text { Sum, Peak) } \\
(8)\end{array}$ & $\begin{array}{c}\text { RMS } \\
(\mathrm{mJy}) \\
(9)\end{array}$ & $\begin{array}{l}\text { Baseline }^{\mathrm{e}} \\
\text { Fit Order } \\
\quad(10)\end{array}$ & $\begin{array}{l}\text { Type } \\
(11)\end{array}$ \\
\hline LARS 01 & $8339 \pm 5$ & $160 \pm 10$ & $180 \pm 16$ & $-55 \pm 5$ & $0.70 \pm 0.07$ & $2.5 \pm 0.3$ & $(3.1,6.5)$ & 1.1 & 2 & $\mathrm{D}$ \\
\hline LARS 02 & $8940 \pm 8$ & $140 \pm 17$ & $150 \pm 26$ & $3 \pm 8$ & $0.70 \pm 0.07$ & $2.8 \pm 0.3$ & $(2.2,2.9)$ & 1.8 & 3 & $\mathrm{D}$ \\
\hline LARS 03 & $9421 \pm 20$ & $310 \pm 39$ & $380 \pm 62$ & $230 \pm 20$ & $1.6 \pm 0.2$ & $6.9 \pm 0.7$ & $(3.1,4.9)$ & 1.2 & 3 & $\mathrm{D}$ \\
\hline LARS 04 & $9740 \pm 16$ & $150 \pm 31$ & $260 \pm 49$ & $-3 \pm 16$ & $1.6 \pm 0.2$ & $7.7 \pm 0.8$ & $(5.1,11)$. & 1.0 & 3 & $\mathrm{~S}$ \\
\hline LARS 05 & $10117 \pm 14$ & $160 \pm 28$ & $170 \pm 44$ & $-10 \pm 14$ & $<0.55$ & $<2.9$ & $(1.3,1.5)$ & 1.1 & 1 & I \\
\hline LARS 06 & $10378 \pm 12$ & $370 \pm 3$ & $390 \pm 5$ & $155 \pm 2$ & $4.4 \pm 0.4$ & 23. \pm 2 & $(9.5,18)$. & 1.1 & 3 & $\mathrm{D}$ \\
\hline LARS 07 & $11315 \pm 16$ & $100 \pm 32$ & $170 \pm 50$ & $-10 \pm 16$ & $0.47 \pm 0.05$ & $3.1 \pm 0.3$ & $(3.3,6.2)$ & 0.86 & 3 & $\mathrm{~S}$ \\
\hline LARS 08 & $11468 \pm 23$ & $310 \pm 47$ & $470 \pm 73$ & $20 \pm 23$ & $3.4 \pm 0.3$ & 22. \pm 2 & $(5.7,11)$. & 0.91 & 3 & I \\
\hline LARS 09 & $14020 \pm 46$ & $270 \pm 92$ & $490 \pm 140$ & $-130 \pm 46$ & $1.2 \pm 0.1$ & 13. \pm 1 & $(2.8,7.3)$ & 0.73 & 3 & $\mathrm{~S}$ \\
\hline LARS 10 & $17284 \pm 69$ & $280 \pm 140$ & $380 \pm 220$ & $70 \pm 69$ & $0.29 \pm 0.03$ & $4.5 \pm 0.5$ & $(1.1,2.1)$ & 0.69 & 3 & $\mathrm{C}$ \\
\hline LARS 11 & $25366 \pm 56$ & $260 \pm 11$ & $290 \pm 18$ & $65 \pm 6$ & $0.75 \pm 0.08$ & 26. \pm 3 & $(2.9,6.4)$ & 0.89 & 3 & D \\
\hline LARS 12 & $(30609)$ & $(264)$ & $(290)$ & $\mathrm{N} / \mathrm{A}$ & $<2.9$ & $<150$ & $\mathrm{~N} / \mathrm{A}$ & 3.4 & 3 & $\mathrm{~N}$ \\
\hline LARS 13 & $(43980)$ & $(264)$ & $(290)$ & $\mathrm{N} / \mathrm{A}$ & $<33$ & $<3800$ & $\mathrm{~N} / \mathrm{A}$ & 38. & 5 & $\mathrm{~N}$ \\
\hline LARS 14 & $(54172)$ & (264) & $(290)$ & $\mathrm{N} / \mathrm{A}$ & $<1.7$ & $<310$ & $\mathrm{~N} / \mathrm{A}$ & 2.0 & 1 & $\mathrm{~N}$ \\
\hline
\end{tabular}

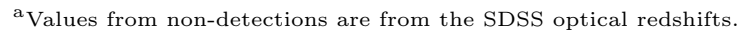

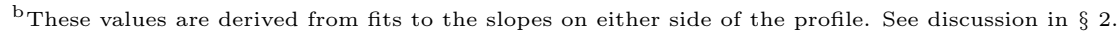

${ }^{\mathrm{c}}$ Assumed linewidths for non detections are the average linewidth of detected sources.

${ }^{\mathrm{d}}$ Upper limits are $1.5 \sigma$ above the local $\mathrm{rms}$ noise over the average $\mathrm{W}_{20}$ value $\left(290 \mathrm{~km} \mathrm{~s}^{-1}\right)$.

e Order of polynomial that was fit to the GBT baseline.

${ }^{f}$ The classification of the profile: 'S' for single-horned, 'D' for double-horned, 'C' for confusion caused by other galaxies, 'I' for an irregular profile, 'N' for no detection. 
Table 3. VLA Observations of the LARS Galaxies

\begin{tabular}{|c|c|c|c|c|c|}
\hline Galaxy & $\begin{array}{l}\text { Observation } \\
\text { Date } \\
(\mathrm{UT}) \\
(2)\end{array}$ & $\begin{array}{l}\text { Primary } \\
\text { Calibrator }\end{array}$ & $\begin{array}{c}\text { Phase } \\
\text { Calibrator } \\
\text { (4) }\end{array}$ & $\begin{array}{l}\text { Flux } \\
\text { Density }^{\mathrm{a}} \\
(\mathrm{Jy}) \\
(5)\end{array}$ & $\begin{array}{c}\text { Integration } \\
\text { Time } \\
\text { (min.) } \\
(6)\end{array}$ \\
\hline LARS 02 & 2013 Apr 3-4 & $0542+498=3 \mathrm{C} 147$ & $\mathrm{~J} 0834+5534$ & $8.28 \pm 0.014$ & 225.9 \\
\hline LARS 02 & 2013 Apr 5-6 & $0542+498=3 \mathrm{C} 147$ & $\mathrm{~J} 0834+5534$ & $8.35 \pm 0.013$ & 225.5 \\
\hline LARS 03 & 2013 Apr 14 & $1331+305=3 \mathrm{C} 286$ & $\mathrm{~J} 1400+6210$ & $4.42 \pm 0.017$ & 200.4 \\
\hline LARS 04 & 2013 Mar 30 & $1331+305=3 \mathrm{C} 286$ & $\mathrm{~J} 1252+5634$ & $2.29 \pm 0.025$ & 120.4 \\
\hline LARS 08 & 2013 Apr 16 & $1331+305=3 \mathrm{C} 286$ & $\mathrm{~J} 1254+1141$ & $0.858 \pm 0.0058$ & 119.8 \\
\hline LARS 09 & 2013 Mar 3-4 & $0542+498=3 \mathrm{C} 147$ & $\mathrm{~J} 0741+3112$ & $2.02 \pm 0.0056$ & 306.2 \\
\hline LARS 09 & 2013 Mar 26-27 & $0542+498=3 \mathrm{C} 147$ & $\mathrm{~J} 0741+3112$ & $2.09 \pm 0.050$ & 281.1 \\
\hline
\end{tabular}

${ }^{a}$ Flux density of phase calibrator derived during calibration. 
Table 4. Observed and Derived VLA H I Properties

\begin{tabular}{|c|c|c|c|c|c|c|c|c|}
\hline $\begin{array}{c}\text { Galaxy } \\
\text { (1) }\end{array}$ & $\begin{array}{c}\mathrm{V}_{\mathrm{sys}} \\
\left(\mathrm{km} \mathrm{s}^{-1}\right) \\
(2)\end{array}$ & $\begin{array}{c}\mathrm{W}_{50^{\mathrm{a}}} \\
\left(\mathrm{km} \mathrm{s}^{-1}\right) \\
(3)\end{array}$ & $\begin{array}{c}\mathrm{W}_{20}^{\mathrm{a}} \\
\left(\mathrm{km} \mathrm{s}^{-1}\right) \\
(4)\end{array}$ & $\begin{array}{l}\text { Velocity Offset } \\
\qquad \begin{array}{c}\left(\mathrm{km} \mathrm{s}^{-1}\right) \\
(5)\end{array}\end{array}$ & $\begin{array}{c}\mathrm{S}_{\mathrm{HI}} \\
\left(\mathrm{Jy} \mathrm{km} \mathrm{s}^{-1}\right) \\
(6)\end{array}$ & $\begin{array}{c}\mathrm{M}_{\mathrm{HI}} \\
\left(10^{9} \mathrm{M}_{\odot}\right) \\
(7)\end{array}$ & $\begin{array}{c}\text { RMS } \\
\left(\mathrm{mJy} / \text { Beam }^{\mathrm{b}}\right) \\
(8)\end{array}$ & $\begin{array}{c}\left\langle\mathrm{N}_{\mathrm{HI}}\right\rangle^{\mathrm{c}} \\
\left(10^{19} \mathrm{~cm}^{-2}\right) \\
(9)\end{array}$ \\
\hline LARS 02 & $8960 \pm 2$ & $140 \pm 4$ & $150 \pm 6$ & $26 \pm 6$ & $0.36 \pm 0.036$ & $1.4 \pm 0.15$ & 0.50 & 2.6 \\
\hline LARS 03 & $9520 \pm 10$ & $170 \pm 20$ & $300 \pm 32$ & $316 \pm 32$ & $2.2 \pm 0.22$ & $7.8 \pm 0.94^{\mathrm{d}}$ & 1.0 & 0.96 \\
\hline LARS 04 & $9760 \pm 2$ & $180 \pm 4$ & $190 \pm 6$ & $15 \pm 6$ & $1.2 \pm 0.12$ & $5.8 \pm 0.58$ & 1.0 & 4.8 \\
\hline LARS 08 & $11450 \pm 2$ & $240 \pm 4$ & $250 \pm 7$ & $-1 \pm 7$ & $1.8 \pm 0.18$ & 12. \pm 1.2 & 1.0 & 2.6 \\
\hline LARS 09 & $14070 \pm 9$ & $310 \pm 18$ & $330 \pm 28$ & $-79 \pm 28$ & $0.60 \pm 0.060$ & $6.2 \pm 0.62$ & 0.50 & 1.2 \\
\hline
\end{tabular}

${ }^{a}$ These values are derived from fits to the slopes on either side of the profile. See discussion in $\S 2$

b The final circular beam sizes of the VLA data presented here are $59^{\prime \prime}, 62^{\prime \prime}, 71^{\prime \prime}, 72^{\prime \prime}$, and $59^{\prime \prime}$, for LARS 02 , LARS 03 , LARS 04 , LARS 08, and LARS 09, respectively.

${ }^{\mathrm{c}}$ Average global value after convolving column density map to the resolution of our beam.

d Mass of main component of galaxy not including tidal tail. 
Table 5. Global UV properties of the LARS Sample Galaxies ${ }^{\mathrm{a}}$

\begin{tabular}{|c|c|c|c|c|c|c|c|c|}
\hline $\begin{array}{c}\text { Galaxy } \\
\text { (1) }\end{array}$ & $\begin{array}{c}\mathrm{L}_{\mathrm{Ly} \alpha} \\
\left(10^{42} \text { cgs. }\right) \\
(2)\end{array}$ & $\begin{array}{c}\mathrm{L}_{H \alpha} \\
\left(10^{42} \text { cgs. }\right) \\
(3)\end{array}$ & $\begin{array}{r}f_{e s c}^{\operatorname{Ly} \alpha} \\
(4)\end{array}$ & $\begin{array}{c}\mathrm{SFR}_{\text {corr }}^{F U V} \\
\left(\mathrm{M}_{\odot} \mathrm{yr}^{-1}\right) \\
(5)\end{array}$ & $\begin{array}{c}\text { Metallicity } \\
(12+\log (\mathrm{O} / \mathrm{H})) \\
(6)\end{array}$ & $\begin{array}{c}\mathrm{W}_{\mathrm{Ly} \alpha} \\
(\AA) \\
(7)\end{array}$ & $\begin{array}{r}\xi_{\mathrm{Ly} \alpha} \\
(8)\end{array}$ & $\begin{array}{c}\mathrm{M}_{*} \\
\left(10^{9} \mathrm{M}_{\odot}\right) \\
(9)\end{array}$ \\
\hline LARS 01 & $0.85 \pm 0.09$ & $0.63 \pm 0.019$ & $0.119 \pm 0.012$ & $3.14 \pm 0.09$ & $8.26 \pm 0.04$ & $33.0 \pm 3.44$ & $3.37_{-0.90}^{+1.1}$ & $6.10 \pm 0.25$ \\
\hline LARS 02 & $0.81 \pm 0.02$ & $0.18 \pm 0.005$ & $0.521 \pm 0.015$ & $1.01 \pm 0.03$ & $8.23 \pm 0.04$ & $81.7 \pm 2.36$ & $2.27_{-0.57}^{+0.83}$ & $2.35 \pm 0.20$ \\
\hline LARS 03 & $0.10 \pm 0.02$ & $0.59 \pm 0.018$ & $0.003 \pm 0.000$ & $2.41 \pm 0.07$ & $8.38 \pm 0.20$ & $16.3 \pm 2.71$ & $0.77_{-0.15}^{+0.10}$ & $20.1 \pm 1.6$ \\
\hline LARS 04 & 0.00 & $0.60 \pm 0.018$ & 0.000 & $3.37 \pm 0.10$ & $8.20 \pm 0.04$ & 0.00 & & $12.9 \pm 0.85$ \\
\hline LARS 05 & $1.11 \pm 0.13$ & $0.51 \pm 0.015$ & $0.174 \pm 0.021$ & $3.23 \pm 0.10$ & $8.13 \pm 0.04$ & $35.9 \pm 4.32$ & $2.61_{-0.60}^{+0.77}$ & $4.27 \pm 0.10$ \\
\hline LARS 06 & 0.00 & $0.08 \pm 0.002$ & 0.000 & $0.62 \pm 0.02$ & $8.08 \pm 0.06$ & 0.00 & & $2.09 \pm 0.15$ \\
\hline LARS 07 & $1.01 \pm 0.01$ & $0.52 \pm 0.016$ & $0.100 \pm 0.001$ & $2.61 \pm 0.08$ & $8.36 \pm 0.05$ & $40.9 \pm 0.41$ & $3.37_{-0.46}^{+0.77}$ & $4.75 \pm 0.11$ \\
\hline LARS 08 & $1.00 \pm 0.07$ & $1.50 \pm 0.045$ & $0.025 \pm 0.002$ & $8.81 \pm 0.26$ & $8.50 \pm 0.15$ & $22.3 \pm 1.59$ & $\begin{array}{r}1.12_{-0.12}^{+0.19} \\
+0.19\end{array}$ & $93.3 \pm 11$ \\
\hline LARS 09 & $0.33 \pm 0.03$ & $2.61 \pm 0.078$ & $0.007 \pm 0.001$ & $15.0 \pm 0.45$ & $8.40 \pm 0.05$ & $3.31 \pm 0.28$ & $>2.85$ & $51.0 \pm 1.4$ \\
\hline LARS 10 & $0.16 \pm 0.05$ & $0.34 \pm 0.010$ & $0.026 \pm 0.008$ & $2.29 \pm 0.07$ & $8.51 \pm 0.14$ & $8.90 \pm 2.82$ & $2.08_{-0.38}^{+0.42}$ & $21.5 \pm 3.2$ \\
\hline LARS 11 & $1.20 \pm 0.20$ & $1.66 \pm 0.050$ & $0.036 \pm 0.006$ & $22.3 \pm 0.67$ & $8.43 \pm 0.30$ & $7.38 \pm 1.22$ & $2.27_{-0.94}^{+0.65}$ & $121 \pm 2.6$ \\
\hline LARS 12 & $0.93 \pm 0.10$ & $1.96 \pm 0.059$ & $0.009 \pm 0.001$ & $13.3 \pm 0.40$ & $8.35 \pm 0.05$ & $8.49 \pm 0.87$ & $3.48_{-0.33}^{+0.46}$ & $7.41 \pm 1.5$ \\
\hline LARS 13 & $0.72 \pm 0.08$ & $2.46 \pm 0.074$ & $0.010 \pm 0.001$ & $18.8 \pm 0.56$ & $8.50 \pm 0.12$ & $6.06 \pm 0.68$ & $1.74_{-0.13}^{+0.06}$ & $59.2 \pm 1.8$ \\
\hline LARS 14 & $4.46 \pm 0.43$ & $1.99 \pm 0.060$ & $0.163 \pm 0.016$ & $11.0 \pm 0.33$ & $8.15 \pm 0.05$ & $39.4 \pm 3.83$ & $3.62_{-0.22}^{+0.82}$ & $1.75 \pm 0.10$ \\
\hline
\end{tabular}

a All values from Hayes et al. (2013), Östlin et al. (2014) and Hayes et al. (2014). 
Table 6. Correlations between H I and global Ly $\alpha$ properties $^{\mathrm{a}}$

\begin{tabular}{|c|c|c|c|c|}
\hline $\begin{array}{c}\text { Ly } \alpha \\
\text { Property } \\
\text { (1) }\end{array}$ & $\begin{array}{c}\mathrm{W}_{50} \\
\rho_{s}^{\mathrm{a}} \\
(2)\end{array}$ & $\begin{array}{c}\mathrm{M}_{H I} \\
\rho_{s}^{\mathrm{a}} \\
(3)\end{array}$ & $\begin{array}{c}\text { Velocity Offset } \\
\rho_{s}{ }^{\mathrm{a}} \\
(5)\end{array}$ & $\begin{array}{c}\mathrm{f}_{g a s} \\
\rho_{s}{ }^{\mathrm{a}} \\
(4)\end{array}$ \\
\hline $\operatorname{Ly} \alpha / \mathrm{H} \alpha$ & $-0.52[0.2]$ & $-0.53[0.06]$ & $-0.083[0.08]$ & $0.47[0.4]$ \\
\hline $\operatorname{Lum}(\operatorname{Ly} \alpha)$ & $-0.27[0.1]$ & $0.05[0.1]$ & $0.17[0.04]$ & $0.00[0.1]$ \\
\hline $\mathrm{H} \alpha / \mathrm{H} \beta$ & $0.67[0.1]$ & $0.75[0.1]$ & $0.50[0.05]$ & $-0.45[0.3]$ \\
\hline $\mathrm{f}_{e s c p}^{\mathrm{Ly} \alpha}$ & $-0.53[0.2]$ & $-0.63[0.1]$ & $-0.15[0.1]$ & $0.40[0.4]$ \\
\hline Ly $\alpha$ EW & $-0.33[0.2]$ & $-0.60[0.1]$ & $0.10[0.1]$ & $0.45[0.3]$ \\
\hline$\xi_{\mathrm{Ly} \alpha}$ & $-0.81[0.1]$ & $-0.45[0.1]$ & $-0.44[0.2]$ & $0.57[0.1]$ \\
\hline $\mathrm{SFR}_{\text {area }}{ }^{-1}$ & $-0.15[0.1]$ & $-0.40[0.2]$ & $-0.067[0.04]$ & $0.43[0.2]$ \\
\hline
\end{tabular}

${ }^{\text {a }}$ Values are for all galaxies with a positive, unconfused detection. Standard deviation values are given in square brackets. 

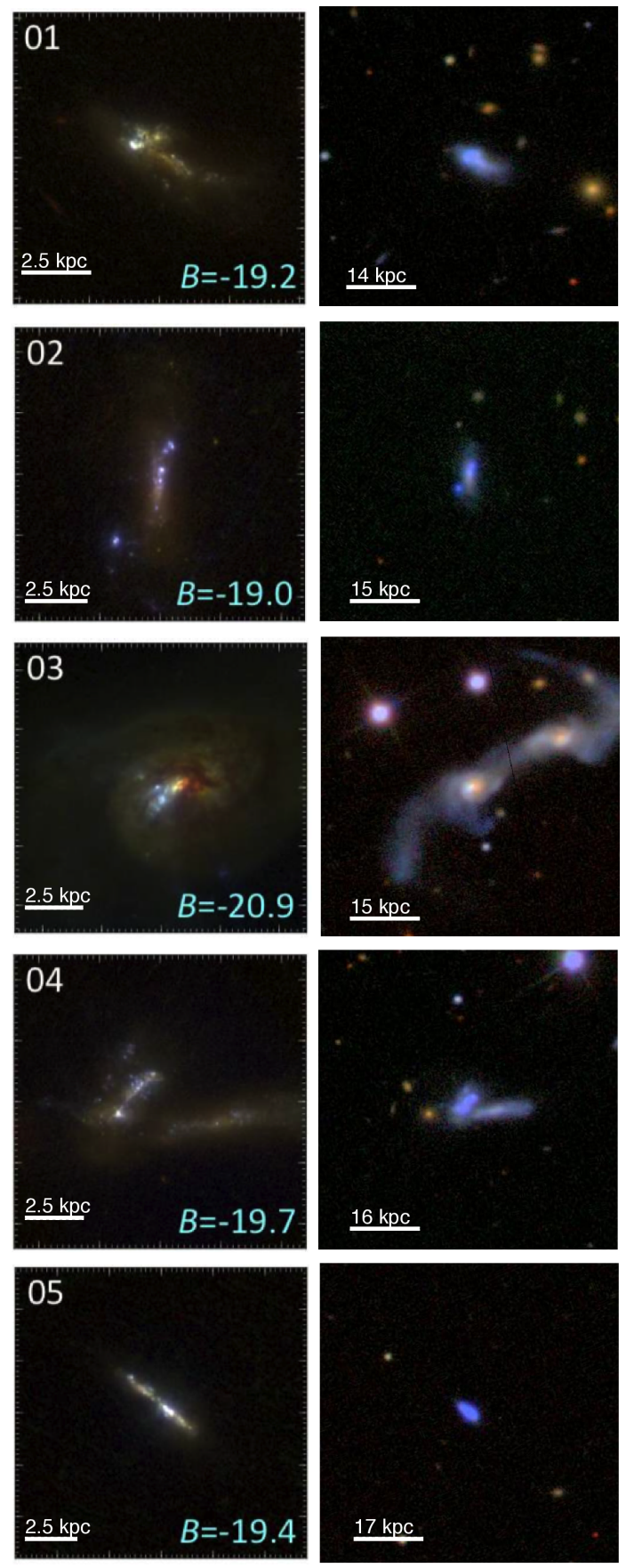

Fig. 1.- Optical and UV views of LARS01 - LARS 05. In the left column are color composite images of the LARS galaxies from HST imaging (red: optical continuum, green: UV continuum, blue: Ly $\alpha$, see Paper II). The number in the upper left panel corresponds to the LARS identification number (e.g., 01 is LARS 01) and each panel in a row is the same LARS galaxy. In the right column are SDSS color images of the LARS target galaxies and the surrounding areas. The fields of view (which are tailored in each frame to show detail) is larger in the right column (1.69' on a side). B band magnitudes, given in blue, were obtained by converting SDSS magnitudes using equations provided by SDSS and found in Jester et al. (2005). These values are corrected for foreground extinction using values from Schlafly \& Finkbeiner (2011). 

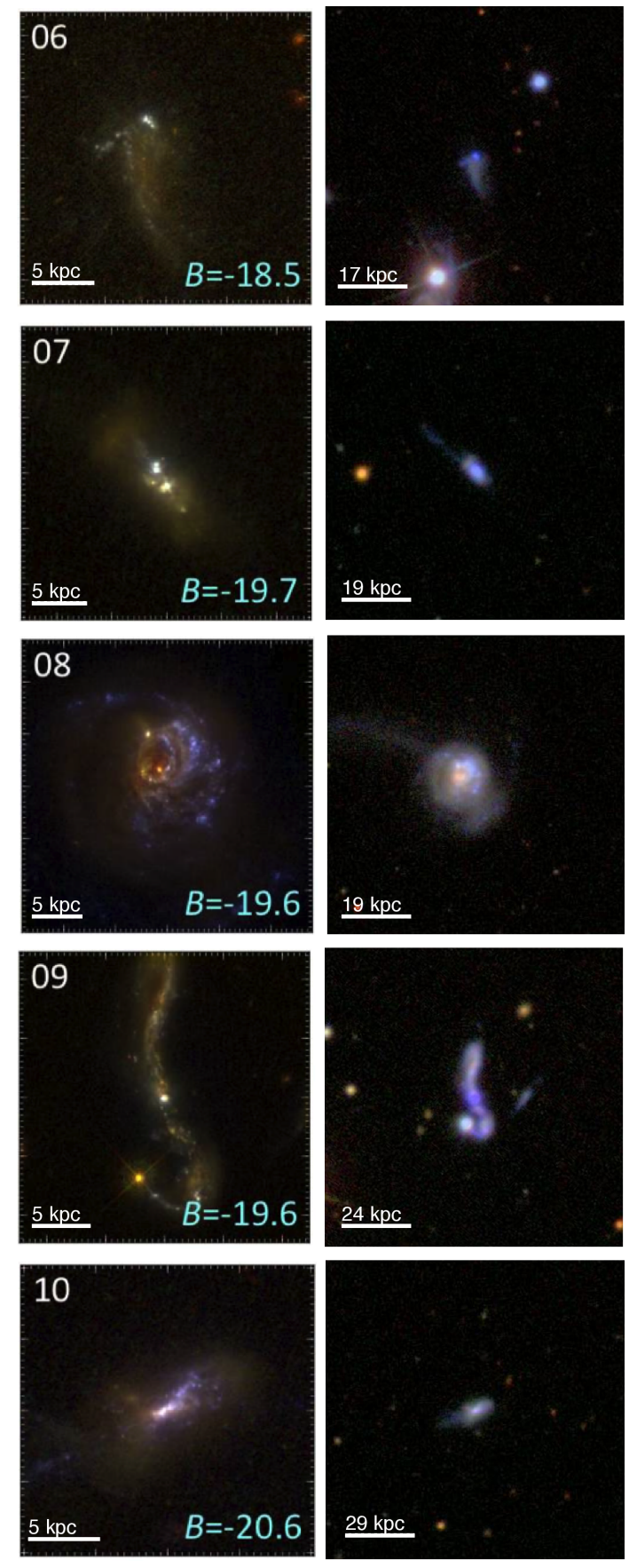

Fig. 2.- Same as Figure 1, for LARS 06 - LARS 10. 


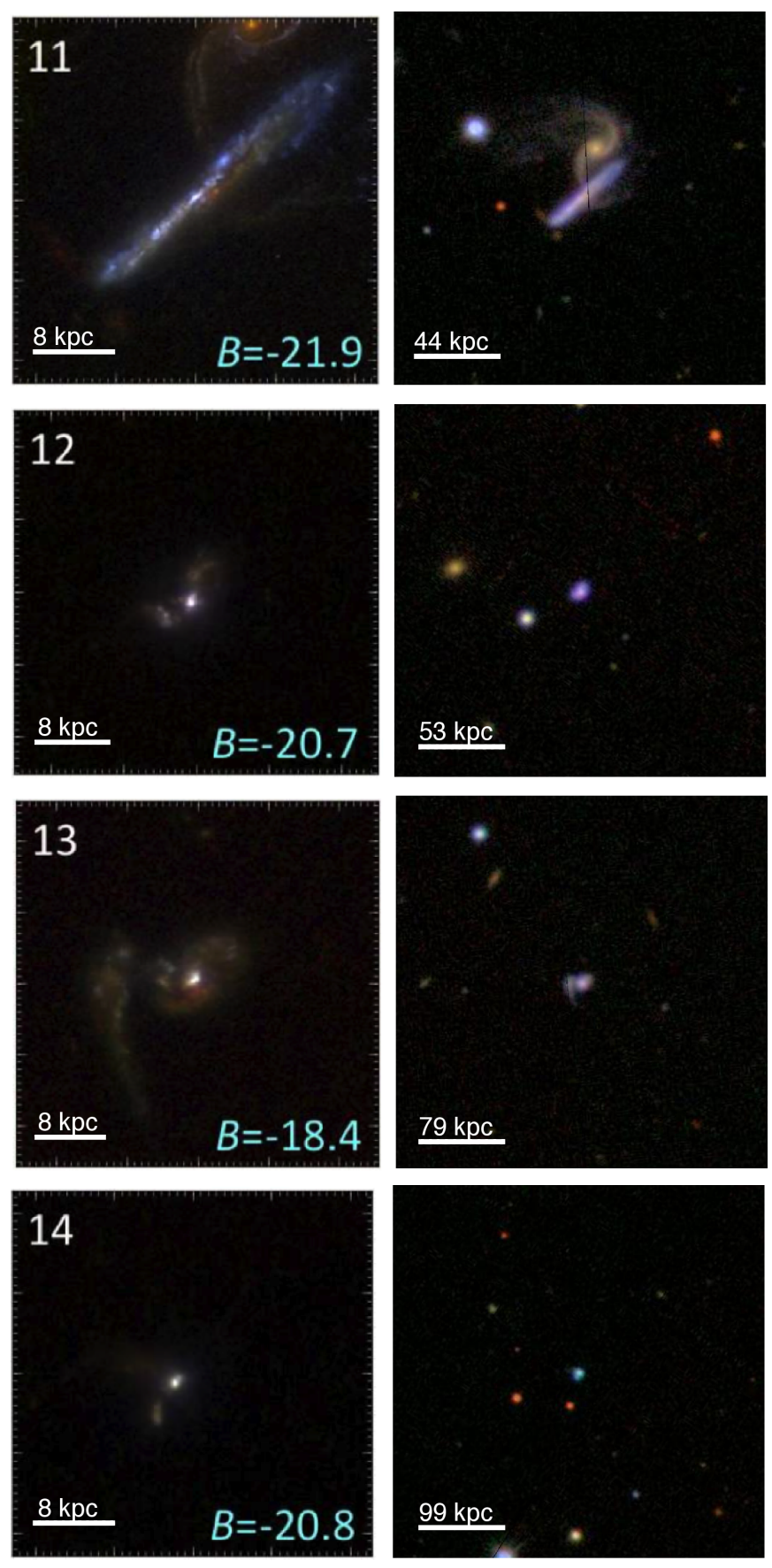

Fig. 3.- Same as Figure 1, for LARS 11 - LARS 14. 

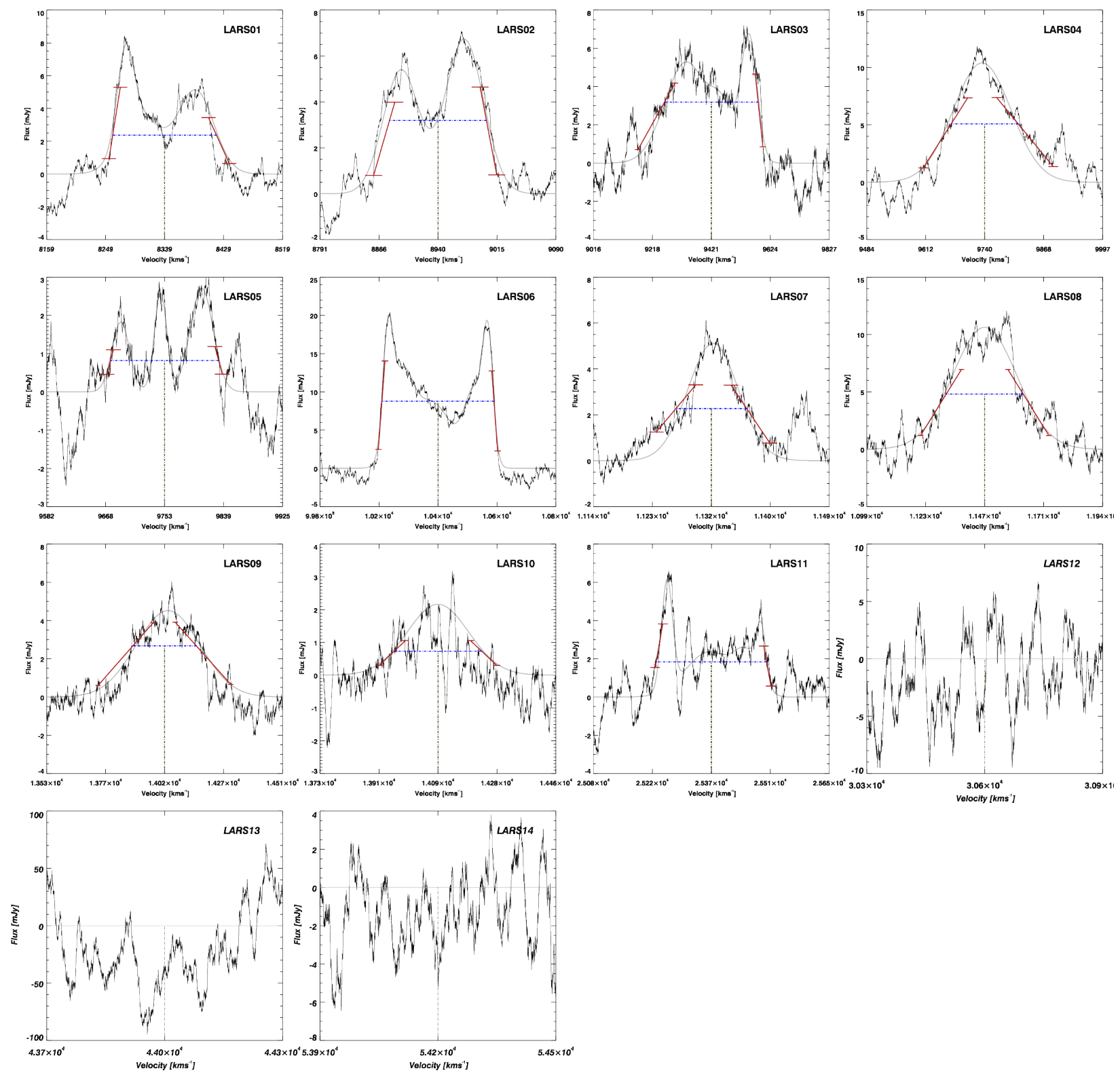

Fig. 4.- GBT H I spectra of LARS Galaxies. We show each smoothed spectrum in black over a gray Gaussian best fit line. The profile is integrated over the area where this best fit rises above the background. The blue dashed line shows the $\mathrm{W}_{50}$ line derisved from the method in Springob et al. (2005) and utilizing the sides of the profile shown by the two red lines on the sides. Refer to $\S 3$ for details. 


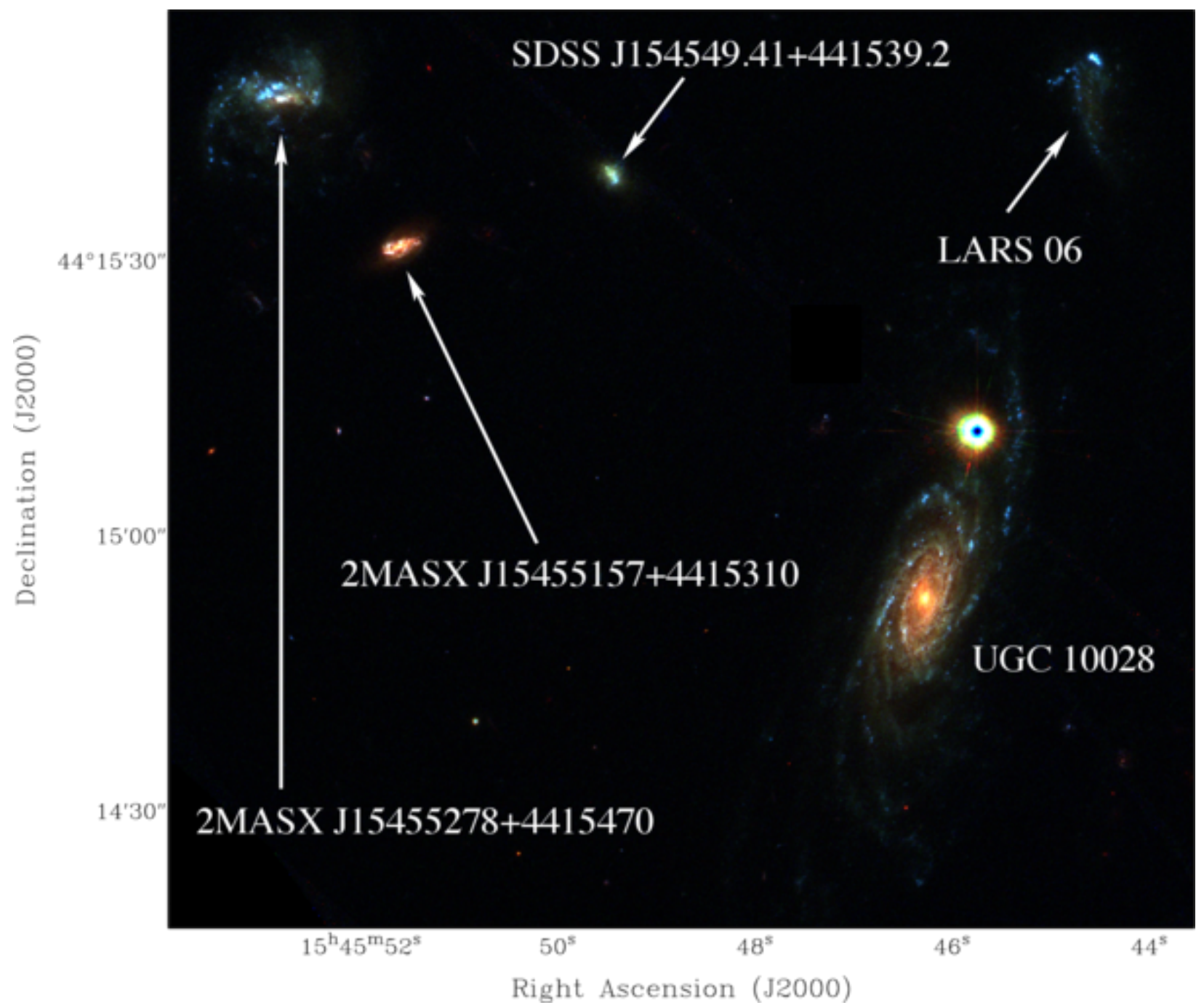

Fig. 5.- HST color composite image (F336W, F435W, and F775W shown as blue, green red, respectively) of the LARS 06 field. The LARS 06 GBT spectrum is potentially confused from at least one of the other labeled sources in the field. Inhomogeneous velocity information is available for all five galaxies: LARS $06\left(\mathrm{~V}_{\mathrm{opt}}=10246 \mathrm{~km} \mathrm{~s}^{-1}\right)$; UGC $10028\left(\mathrm{~V}_{\mathrm{opt}}=10399\right.$ $\left.\mathrm{km} \mathrm{s}^{-1}\right)$; 2MASX J15455278+4415470 $\left(\mathrm{V}_{\text {opt }}=11984 \mathrm{~km} \mathrm{~s}^{-1}\right) ; 2$ MASX J15455157+4415310 $(\mathrm{SDSS}$ RF method photometric redshift $=0.100 \pm 0.0291) ;$ SDSS J154549.41+441539.2 (SDSS RF method photometric redshift $=0.043 \pm 0.0174$ ). Based on this information, LARS 06 appears to be at essentially the same distance as UGC 10028; the other three sources appear to be in the background. Spectroscopic follow-up will be required to determine the absolute velocities of 2MASX J15455278+4415470 and 2MASX J15455157+4415310 (for example, note that the SDSS RF method photometric redshift of UGC 10028 is $0.071 \pm 0.0261$ ). The field of view is much smaller than the GBT primary beam; VLA observations of this complex field will be able to localize the neutral gas components of each system. 


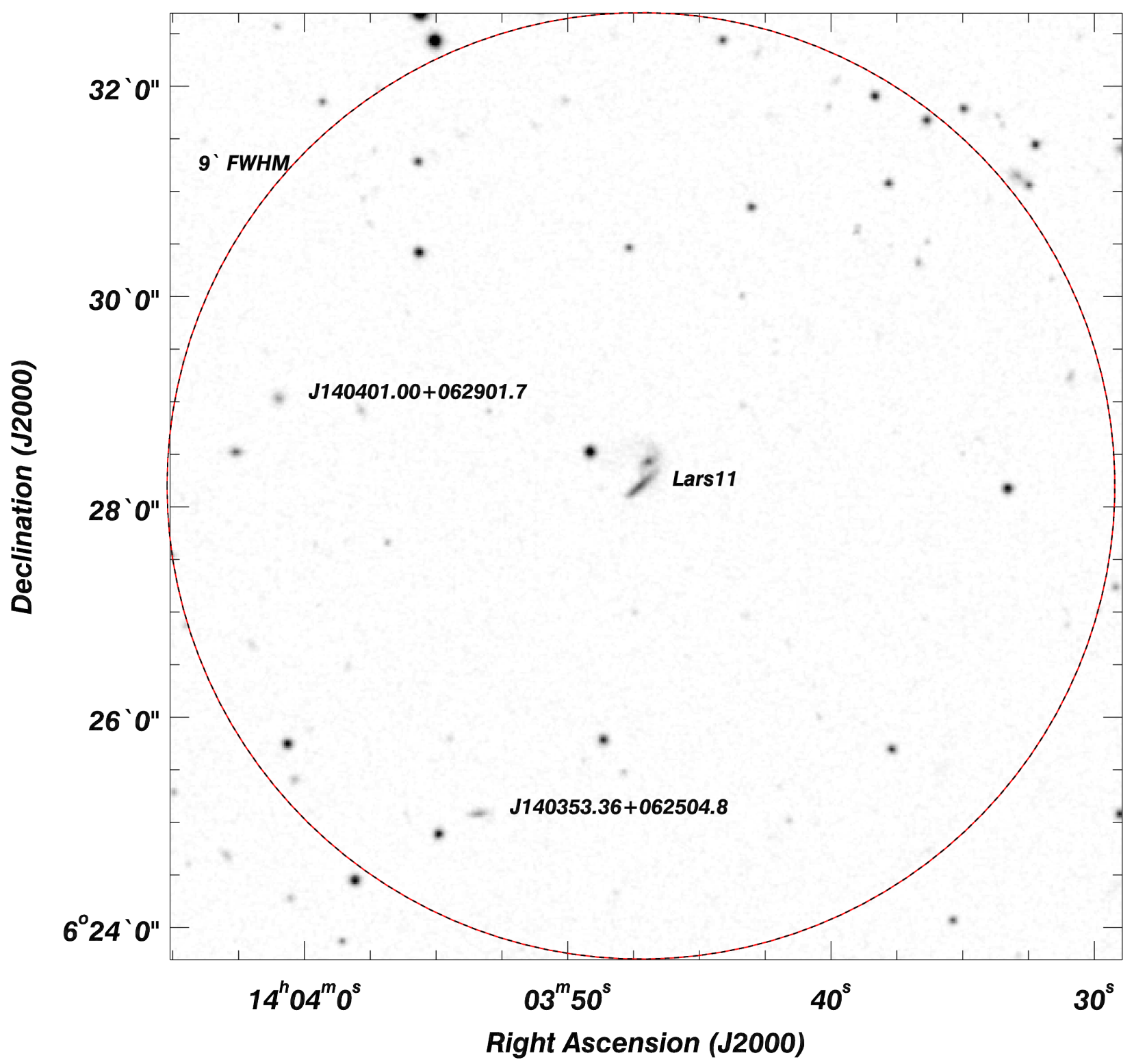

Fig. 6.- Digitized Sky Survey image of the LARS 11 field. The red circle denotes the 8' GBT primary beam; labels denote the target source as well as possible contaminating galaxies at similar velocities within the primary beam. 


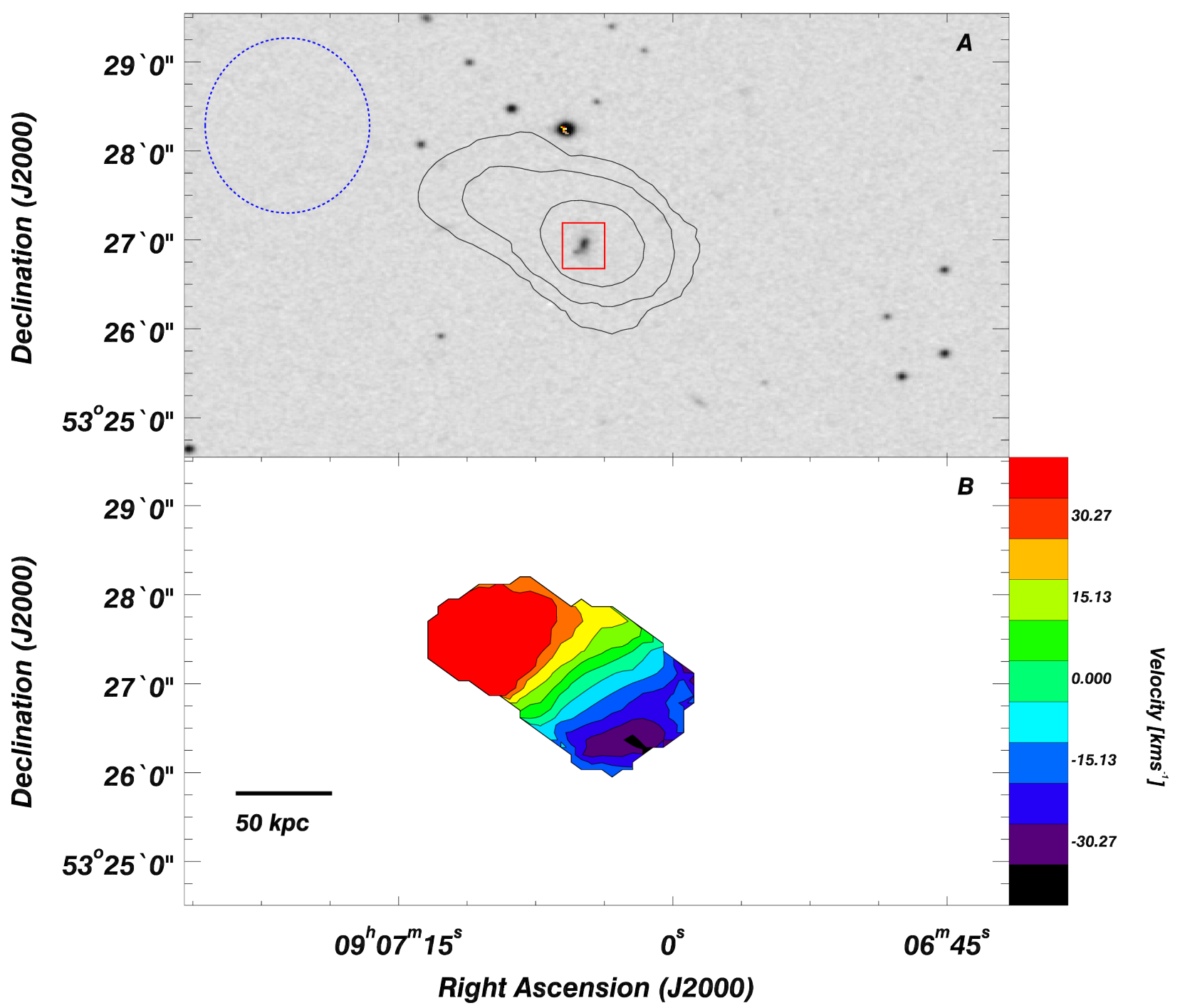

Fig. 7.- H I and optical comparison of LARS 02. Panel (a) shows a Digitized Sky Survey image, overlaid with contours of $\mathrm{H}$ I surface density at levels of $(0.65,1.3,2.6,5.2,10.4,20.8) \times 10^{19}$ $\mathrm{cm}^{-2}$. The beam size $\left(59^{\prime \prime}\right)$ is shown in the upper left, and the approximate location and size of the $14 \mathrm{kpc}$ x $14 \mathrm{kpc}$ HST UV imaging is shown by a red square (see images and discussion in Östlin et al. 2014 and Hayes et al. 2014). Panel (b) shows the H I intensity-weighted velocity field. 


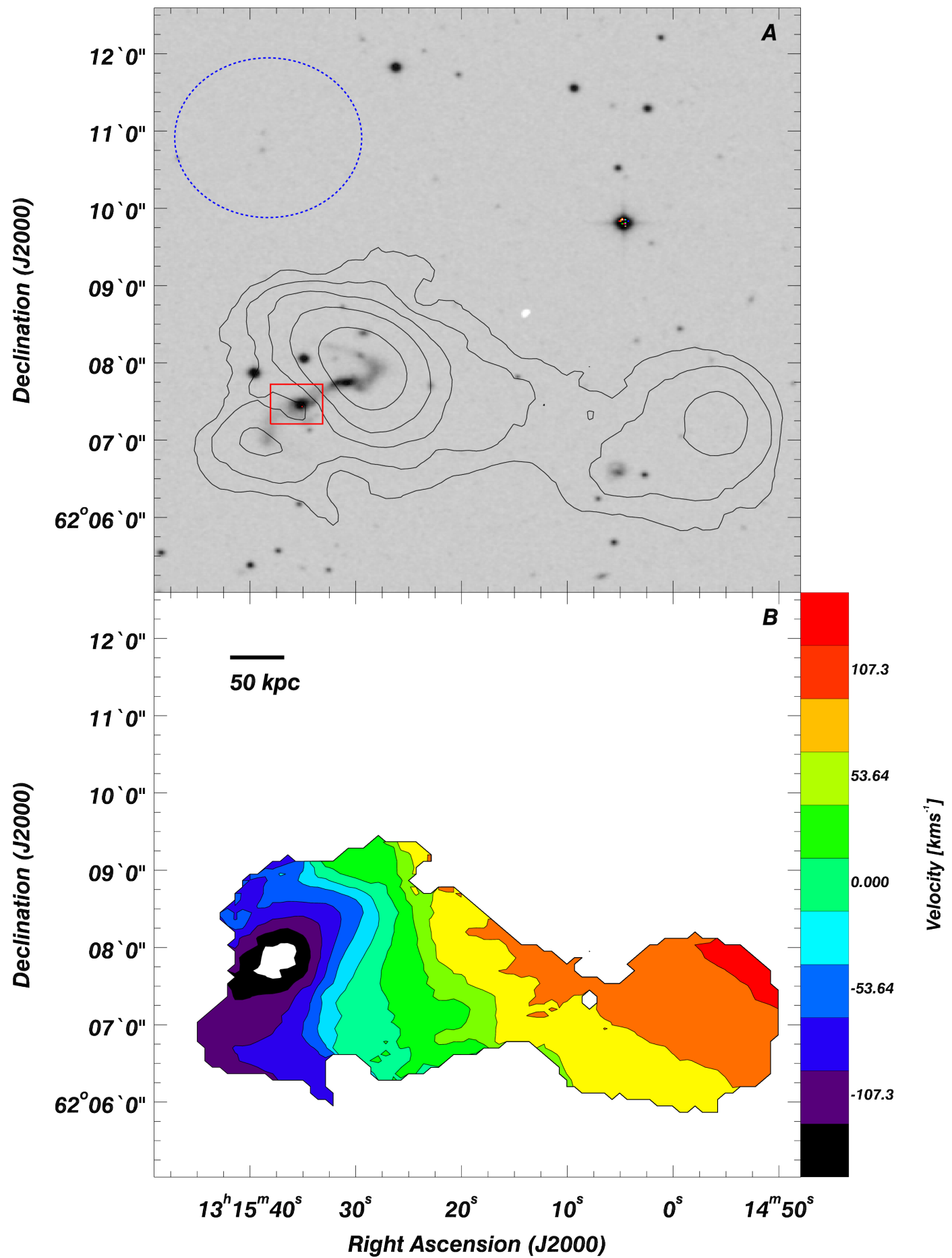

Fig. 8.- H I and optical comparison of LARS 03. Panel (a) shows a Digitized Sky Survey image, overlaid with contours of H I surface density at levels of $(0.65,1.3,2.6,5.2,10.4,20.8) \times 10^{19}$ $\mathrm{cm}^{-2}$. The beam size $\left(62^{\prime \prime}\right)$ is shown in the upper left, and the approximate location and size of the $13 \mathrm{kpc} \times 13 \mathrm{kpc}$ HST UV imaging is shown by a red square (see images and discussion in Östlin et al. 2014 and Hayes et al. 2014). Panel (b) shows the H I intensity-weighted velocity field. 


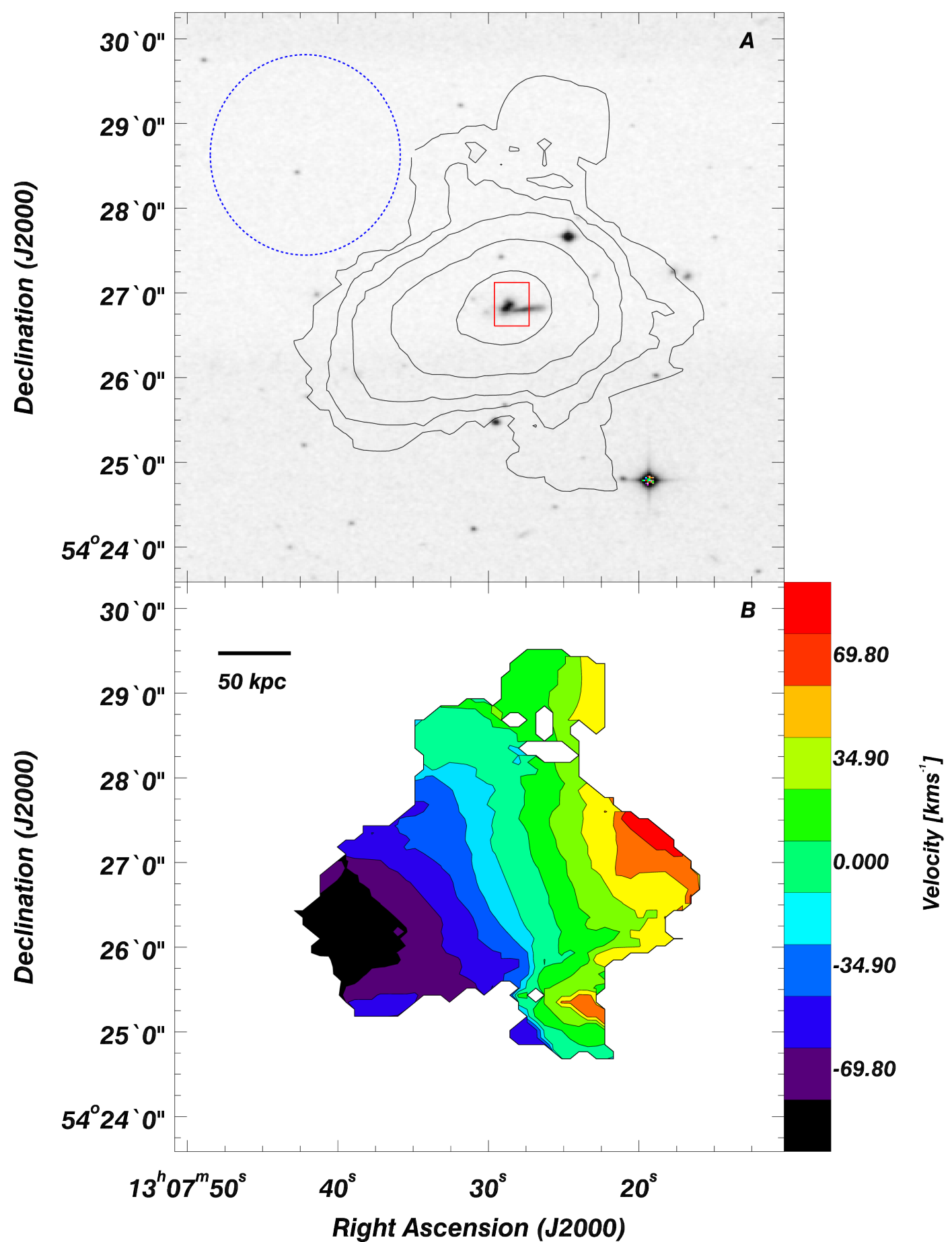

Fig. 9.- H I and optical comparison of LARS 04. Panel (a) shows a Digitized Sky Survey image, overlaid with contours of H I surface density at levels of $(0.65,1.3,2.6,5.2,10.4,20.8) \times 10^{19}$ $\mathrm{cm}^{-2}$. The beam size $\left(71^{\prime \prime}\right)$ is shown in the upper left, and the approximate location and size of the $18 \mathrm{kpc}$ x $18 \mathrm{kpc}$ HST UV imaging is shown by a red square (see images and discussion in Östlin et al. 2014 and Hayes et al. 2014). Panel (b) shows the H I intensity-weighted velocity field. 


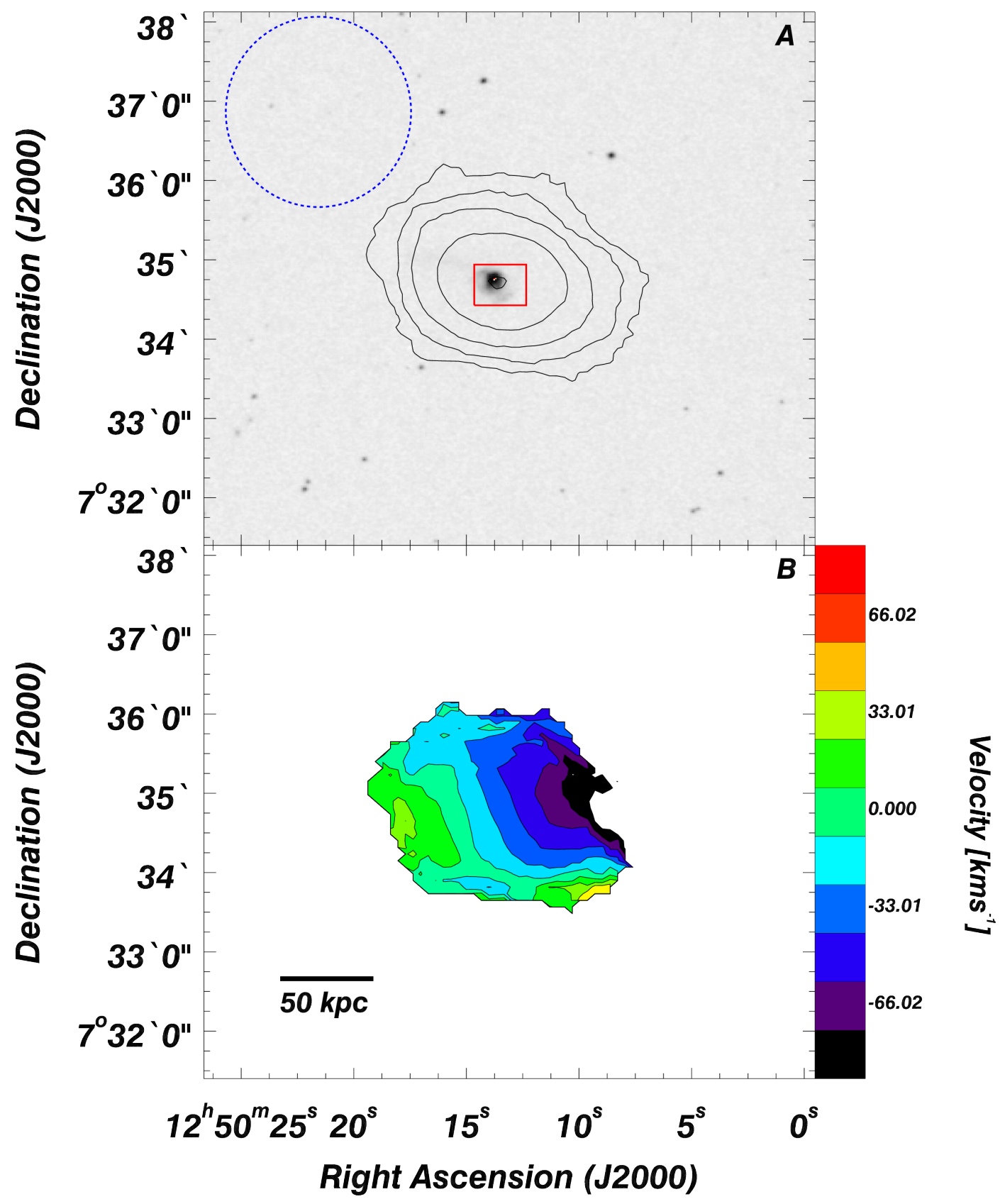

Fig. 10.- H I and optical comparison of LARS08. Panel (a) shows a Digitized Sky Survey image, overlaid with contours of H I surface density at levels of $(0.65,1.3,2.6,5.2,10.4,20.8) \times 10^{19} \mathrm{~cm}^{-2}$. The beam size $\left(72^{\prime \prime}\right)$ is shown in the upper left, and the approximate location and size of the $20 \mathrm{kpc} \times 20 \mathrm{kpc}$ HST UV imaging is shown by a red square (see images and discussion in Östlin et al. 2014 and Hayes et al. 2014). Panel (b) shows the H I intensity-weighted velocity field. 


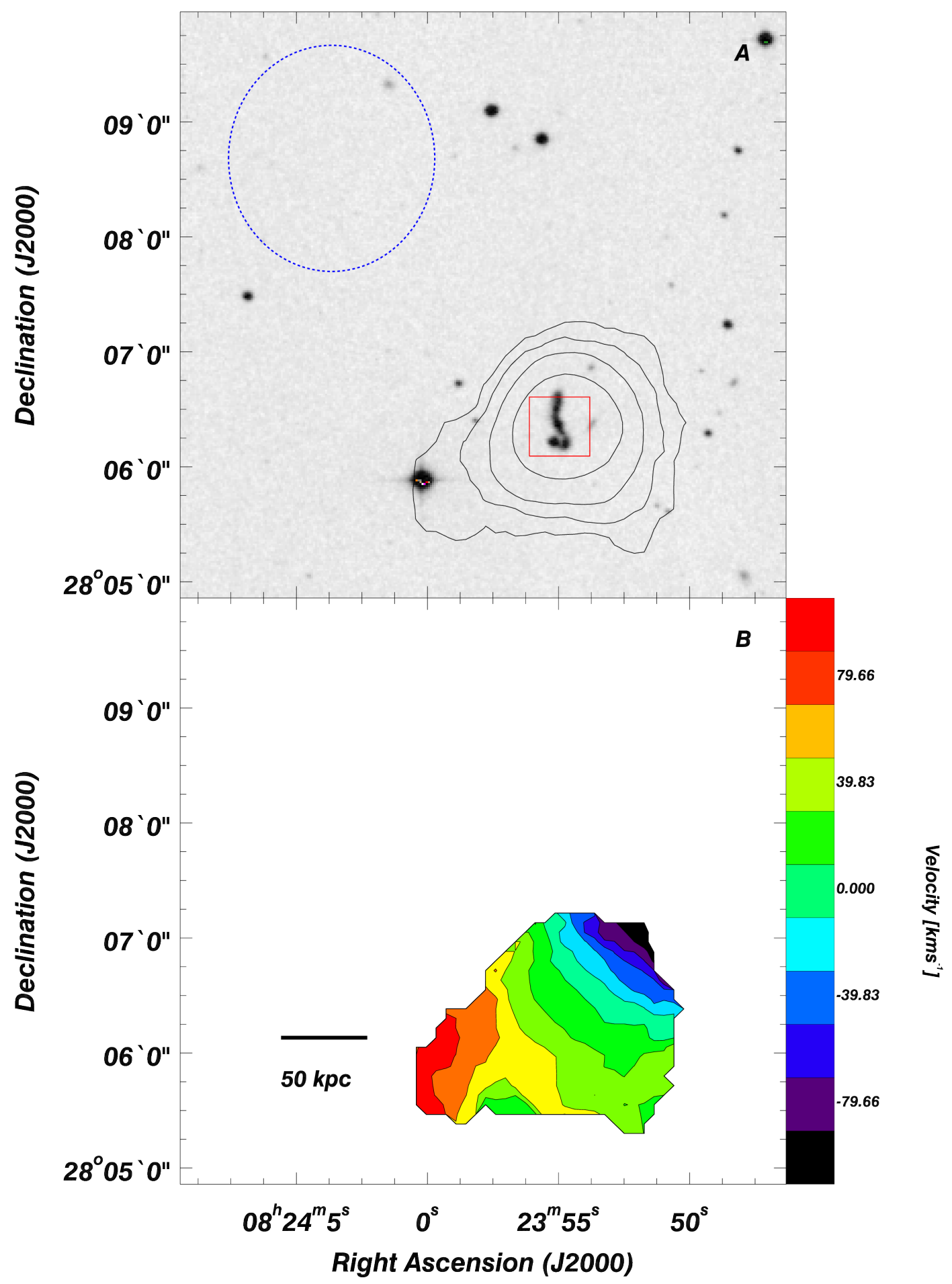

Fig. 11.- H I and optical comparison of LARS 09. Panel (a) shows a Digitized Sky Survey image, overlaid with contours of H I surface density at levels of $(0.65,1.3,2.6,5.2,10.4,20.8) \times 10^{19} \mathrm{~cm}^{-2}$. The beam size $\left(59^{\prime \prime}\right)$ is shown in the upper left, and the approximate location and size of the $26 \mathrm{kpc} \times 26 \mathrm{kpc}$ HST UV imaging is shown by a red square (see images and discussion in Östlin et al. 2014 and Hayes et al. 2014). Panel (b) shows the H I intensity-weighted velocity field. 

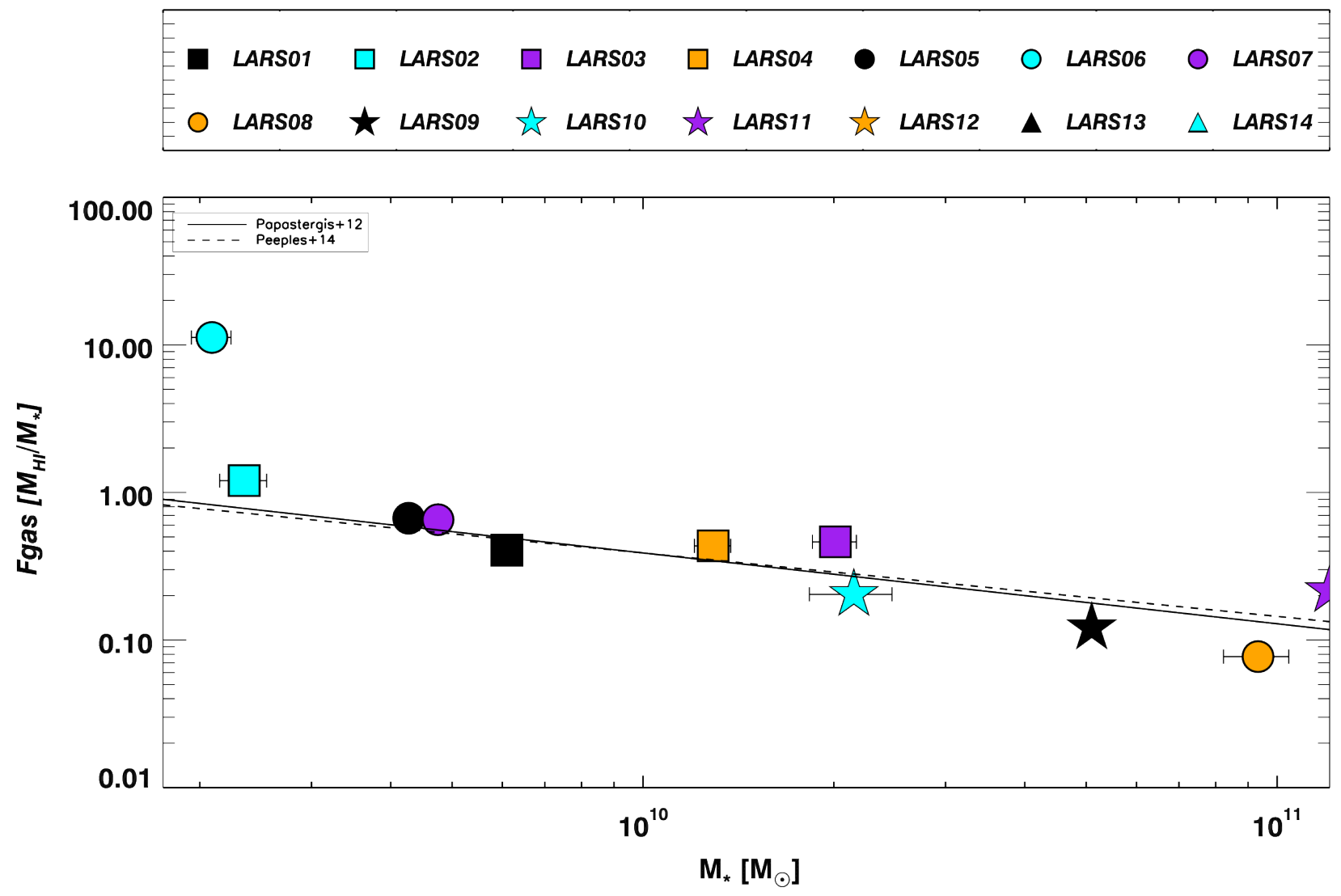

Fig. 12. - Gas fraction versus total stellar mass, where $\mathrm{M}_{\mathrm{HI}}$ is the mass of $\mathrm{H} \mathrm{I}$ and $\mathrm{M}_{*}$ is derived from 2-component SED modeling to HST data (see Hayes et al. 2014). The outlier is LARS 06, whose H I mass measurement is almost certainly contaminated by the nearby field spiral UGC 10028 (see Figure 5). We also show the correlations derived by Papastergis et al. (2012) and Peeples et al. (2014). It should be noted that these lines also take into account molecular gas, whereas our galaxies do not have molecular gas values. 

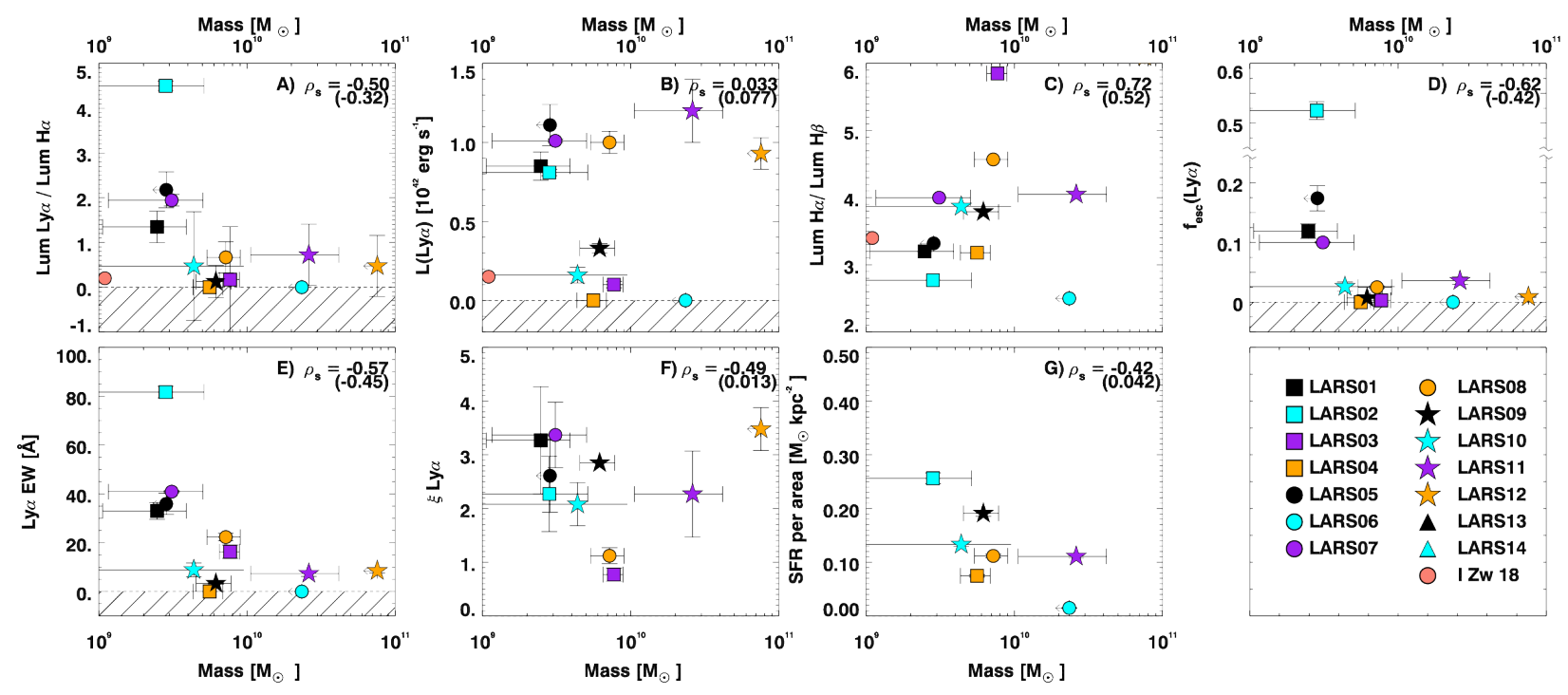

Fig. 13.- Comparisons of global H I mass with the seven global properties derived from HST imaging, including Ly $\alpha / \mathrm{H}_{\alpha}, \mathrm{L}_{\mathrm{L} \alpha}, \mathrm{H}_{\alpha} / \mathrm{H}_{\beta}, f_{\mathrm{esc}}^{\mathrm{Ly} \alpha}$, Ly $\alpha \mathrm{EW}, \mathrm{Ly} \alpha \xi$, and UV SFR/R $\mathrm{R}_{P 20}^{\mathrm{Ly} \alpha}$. When available, we use the VLA derived values for H I mass, which are generally lower than the GBT derived values. The Spearman $\rho_{s}$ correlation coefficient, which quantifies possible monotonic correlations between properties, is shown in each panel. We compare these results with data from another local Ly $\alpha$ emitter, IRAS 08339+6517. The symbols represent the different galaxies and are shown in the legend in the final panel. The same symbols are used throughout the rest of the comparison plots. 

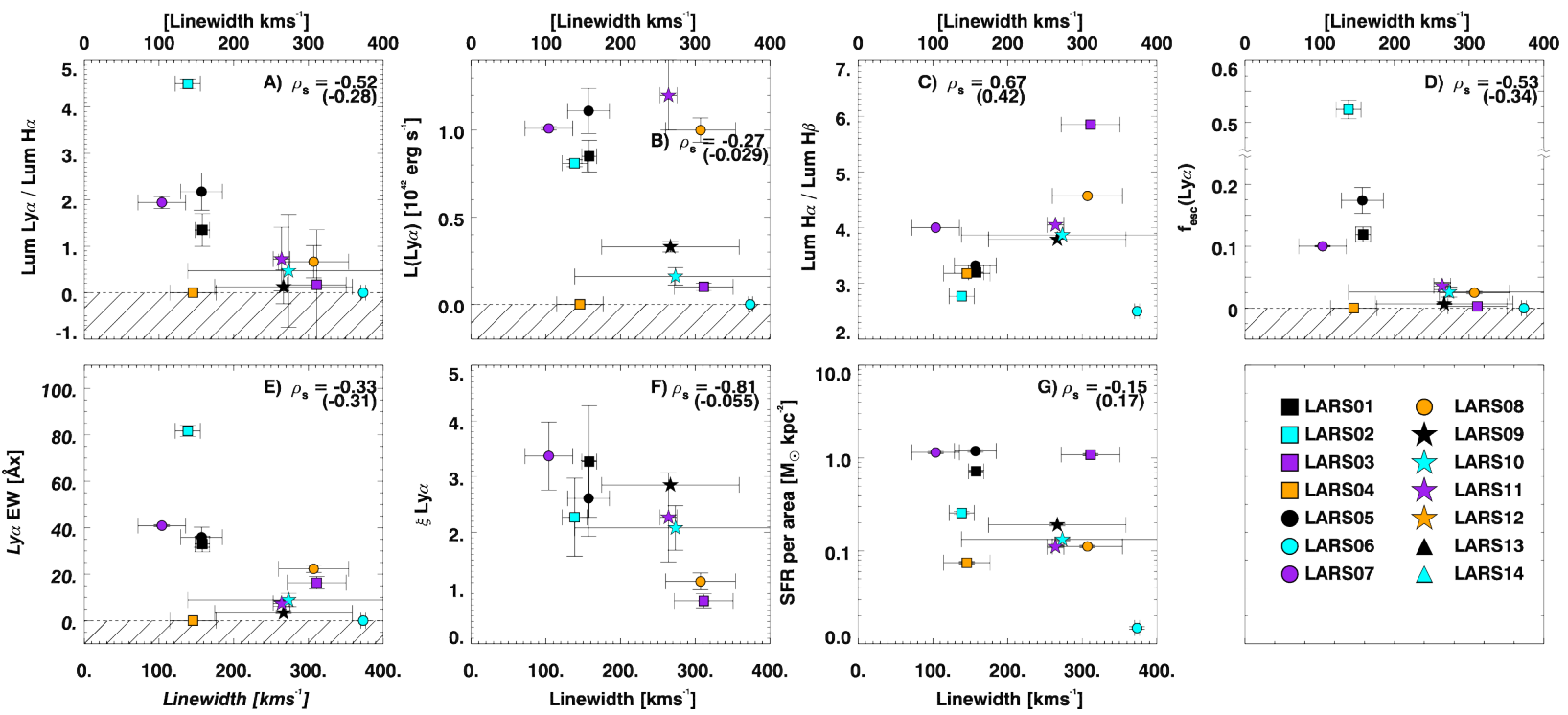

Fig. 14. - Comparisons of global H I line width at $50 \%$ of maximum (see column 3 of Table 2 ) with the seven global properties derived from HST imaging, including $\mathrm{Ly} \alpha / \mathrm{H}_{\alpha}, \mathrm{L}_{\mathrm{L} \alpha}, \mathrm{H}_{\alpha} / \mathrm{H}_{\beta}$, $f_{\mathrm{esc}}^{\mathrm{Ly} \alpha}, \operatorname{Ly} \alpha \mathrm{EW}, \operatorname{Ly} \alpha \xi$, and UV SFR/R $\mathrm{R}_{P 20}^{\mathrm{Ly} \alpha}$. The Spearman $\rho_{s}$ correlation coefficient is shown in each panel for the subsample of galaxies with unconfused detections and, in parenthesis, all fourteen galaxies. The symbols are the same as in Figure 13. 

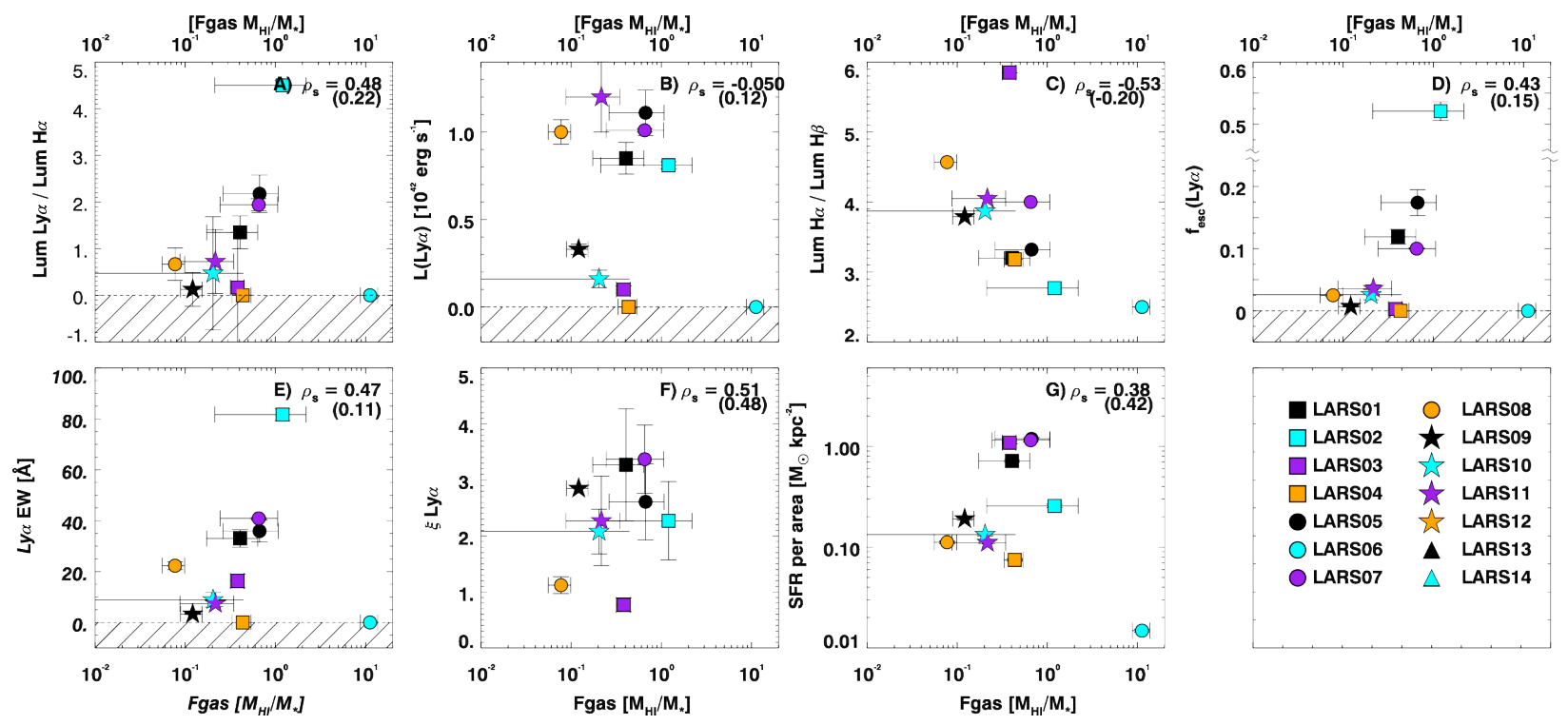

Fig. 15. - Comparisons between the gas fraction $\left(\mathrm{M}_{H_{I}} / \mathrm{M}_{*}\right)$ and the seven global properties derived from HST imaging, including $\mathrm{Ly} \alpha / \mathrm{H}_{\alpha}, \mathrm{L}_{\mathrm{L} \alpha}, \mathrm{H}_{\alpha} / \mathrm{H}_{\beta}, f_{\mathrm{esc}}^{\mathrm{Ly} \alpha}$, Ly $\alpha \mathrm{EW}$, Ly $\alpha \xi$, and UV $\mathrm{SFR} / \mathrm{R}_{P 20}^{\mathrm{Ly} \alpha}$. The symbols and correlation coefficients are plotted the same as in Figure 13. 

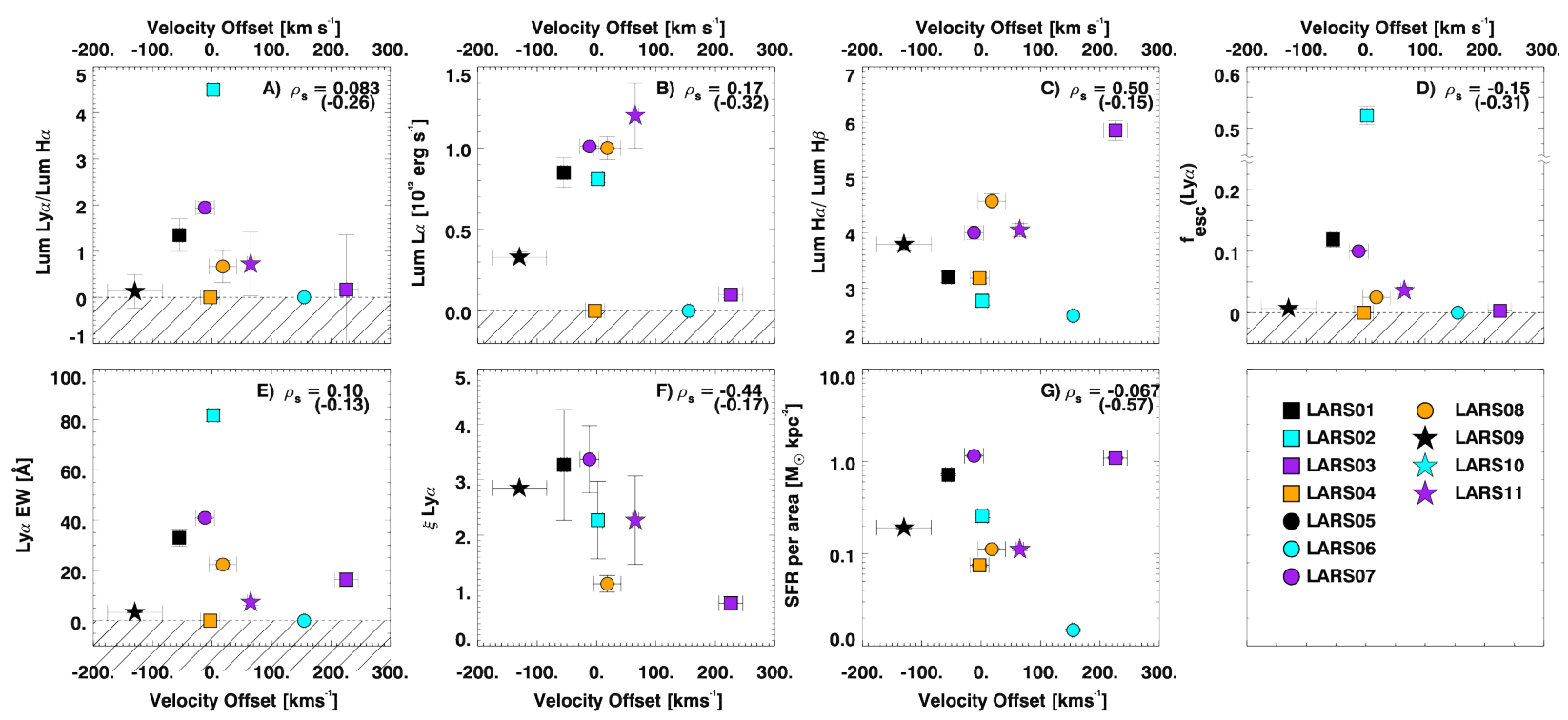

Fig. 16. - Comparisons between the velocity offset between optical and H I derived systemic velocities and the seven global properties derived from HST imaging, including $\mathrm{Ly} \alpha / \mathrm{H}_{\alpha}, \mathrm{L}_{\mathrm{L} \alpha}$, $\mathrm{H}_{\alpha} / \mathrm{H}_{\beta}, f_{\mathrm{esc}}^{\mathrm{Ly} \alpha}$, Ly $\alpha \mathrm{EW}, \mathrm{Ly} \alpha \xi$, and UV SFR/R $\mathrm{R}_{P 20}^{\mathrm{Ly} \alpha}$. The symbols and correlation coefficients are plotted the same as in Figure 13 . 